\title{
A VARIATIONAL APPROACH FOR COMPACT HOMOGENEOUS EINSTEIN MANIFOLDS
}

\author{
CHRISTOPH BÖHM, MCKENZIE WANG, AND WOLFGANG ZILLER
}

Einstein metrics of volume 1 on a closed manifold can be characterized variationally as the critical points of the Hilbert action [Hi], which associates to each Riemannian metric of volume 1 the integral of its scalar curvature. As is well-known, the gradient vector of the Hilbert action with respect to the natural $L^{2}$ metric is precisely the negative of the traceless Ricci tensor. Consequently, if $G$ is a compact group of diffeomorphisms of the manifold, then unit volume $G$-invariant Einstein metrics are the critical points of the restriction of the Hilbert action to the subspace of unit volume $G$-invariant metrics.

In this paper we shall exploit this principle to study homogeneous Einstein metrics from a variational viewpoint. Suppose $G$ is a compact Lie group and $H$ is a closed subgroup so that $M=G / H$ is connected. The restriction of the Hilbert action to the finite-dimensional space $\mathcal{M}_{1}^{G}$ of unit volume $G$-invariant metrics is then just the scalar curvature function $S: \mathcal{M}_{1}^{G} \rightarrow \mathbb{R}$ that associates to each invariant metric its scalar curvature (a constant), and $G$-invariant Einstein metrics are precisely the critical points of $S$.

In the homogeneous case, the Einstein equations are algebraic, and many authors (see, e.g., references in [Bes], [Wa]) have made substantial progress in solving these equations, often explicitly. In particular, classifications of compact homogeneous Einstein manifolds in low dimensions are known (cf. [Je1], [ADF], [NR], [Ni]). However, in higher dimensions examples exist where $G / H$ carries no $G$-invariant Einstein metric [WZ2]. Moreover, for a generic homogeneous space, it is hard to describe the coefficients of the Einstein equations uniformly, and the task of finding at least one real solution presents a serious difficulty. One way to overcome these problems is to pursue a global variational approach based on the scalar curvature function $S$.

Such an approach was first used by G. Jensen to study left-invariant Einstein metrics on compact Lie groups [Je2]. It was again taken up by the last two authors in [WZ2], where it was shown that $S$ is bounded from above and proper iff $\mathfrak{h}$ is a maximal Lie subalgebra of $\mathfrak{g}$. In this case, $S$ has a maximum point, which must be a $G$-invariant Einstein metric.

Hence, further existence theorems for homogeneous Einstein metrics by the above variational approach will involve searching for general saddle points of $S$ when it is unbounded both from above and from below. The first important step in such a program is to show that $S$ satisfies some form of the Palais-Smale condition, which says that if $S\left(g_{i}\right)$ is bounded and $\left\|(\operatorname{grad} S)_{g_{i}}\right\|$ converges to 0 in an appropriate norm, then there exists a convergent subsequence which converges to a critical point (in an appropriate topology). For \|\| we shall use the $L^{2}$ norm, which by homogeneity reduces to $\|h\|_{g}$ for $h$ a tangent vector at $g \in \mathcal{M}_{1}^{G}$.

Our first main result is:

Theorem A. Let $M=G / H$ be a compact homogeneous space. Then for every $\epsilon>0$, the Palais-Smale condition is satisfied on the subset $\left(\mathcal{M}_{1}^{G}\right)_{\epsilon}=\left\{g \in \mathcal{M}_{1}^{G} \mid S(g) \geq \epsilon\right\}$.

The second author was partly supported by NSERC grant No. OPG0009421 and the third author was supported in part by a grant from the National Science Foundation and by the Francis J. Carey Term Chair. 
The assumption $\epsilon>0$ is not a serious restriction, since compact homogeneous spaces cannot carry homogeneous Einstein metrics with negative scalar curvature (Bochner's theorem [Bo]), and a Ricci-flat homogeneous metric must be flat (Alekseevsky-Kimel'fel'd [AK]), which in turn implies that $\mathrm{M}$ is a torus. Hence we are only interested in Einstein metrics with positive scalar curvature, which all lie in $\left(\mathcal{M}_{1}^{G}\right)_{\epsilon}$ for some $\epsilon>0$.

On the other hand, sometimes there exist sequences $g_{i}$ with $S\left(g_{i}\right) \rightarrow 0$ and $-(\operatorname{grad} S)_{g_{i}}=$ $\operatorname{Ric}^{0}\left(g_{i}\right)=\operatorname{Ric}\left(g_{i}\right)-\frac{S_{g_{i}}}{n} g_{i} \rightarrow 0$. They will not have a convergent subsequence if $G / H$ is not a torus. One obtains such a sequence, if there exists a group $K$ with $H \subset K \subset G$ and $K / H$ a torus, by shrinking the fibres in the fibration $G / H \rightarrow G / K$ to a point. We will essentially show that if no such $K$ exists, the Palais-Smale condition is satisfied on all of $\mathcal{M}_{1}^{G}$ (cf. Theorem 2.1). We should point out though that the Palais-Smale condition of Theorem A for the Hilbert action fails in general, even in the cohomogeneity one case. Indeed, in [Bö1] a sequence of cohomogeneity one Einstein metrics is constructed whose scalar curvature converges to a positive constant, but the metrics do not have a convergent subsequence.

To prove Theorem A, one observes first that if $S\left(g_{i}\right) \rightarrow \lambda>0$ and $\operatorname{Ric}^{0}\left(g_{i}\right) \rightarrow 0$ then we obtain a uniform positive lower bound for the Ricci curvature and hence also for the volumes of unit metric balls. One then uses the deep theory developed by Cheeger and Colding in [CC] to obtain a gauge-transformed subsequence which converges in the $C^{1, \alpha}$ topology to an Einstein metric. Finally, one analyzes the limit using equivariant Gromov-Hausdorff convergence and classical results about transformation groups. It is somewhat surprising that although $S: \mathcal{N}_{1}^{G} \rightarrow \mathbb{R}$ is an algebraic function, a direct proof of Theorem A seems to be rather difficult.

An important consequence of Theorem A is the following compactness result, which solves a fundamental open problem in this subject:

Corollary. Let $G / H$ be a compact homogeneous space with finite fundamental group. Then the moduli space of G-invariant Einstein metrics has only finitely many components, each one of which is compact.

Notice that the assumption that the fundamental group is finite is again not a restriction, since the existence of an Einstein metric with positive scalar curvature implies that the fundamental group is finite by Bonnet-Myers.

The advantage of our geometric proof of Theorem A is that we obtain convergence even when $g_{i}$ are merely assumed to be homogeneous, i.e., when the transitive action or even $G$ can change. Hence we also get compactness for the set of all homogeneous Einstein metrics, as long as we fix a positive lower bound for the Einstein constant. This result is optimal, since $S^{2} \times S^{2 n+1}$ admits infinitely many inequivalent transitive actions, and each one has a unique invariant Einstein metric of volume one whose scalar curvature converges to 0 [WZ3].

In view of the above compactness result for the homogeneous Einstein moduli space, one of the most important open questions is whether in some cases the components of this moduli space can have positive dimension after dividing by the action of the natural gauge group $N(H) / H$ on $\mathcal{M}_{1}^{G}$. So far, on all compact homogeneous spaces $G / H$ with finite fundamental group, up to isometry, only finitely many $G$-invariant Einstein metrics are known.

We propose the following

Finiteness ConjeCture. If $G / H$ is a compact homogeneous space whose isotropy representation consists of pairwise inequivalent irreducible summands, e.g., when rank $G=\operatorname{rank} H$, then the algebraic Einstein equations have only finitely many real solutions. 
Under this assumption, the identity component of $N(H) / H$ acts trivially on $\mathcal{M}_{1}^{G}$, and hence we indeed expect only finitely many real solutions.

To give the reader a feeling for the Einstein equations in this special case, we fix a bi-invariant background metric $Q$, so that any $G$-invariant metric is given by $\sum_{i=1}^{\ell} x_{i} Q \mid \mathfrak{m}_{i}$, where $\mathfrak{m}_{i}$ are the irreducible summands of the isotropy representation. The metric is then Einstein with Einstein constant $\lambda$ iff

$$
\frac{b_{i}}{2 x_{i}}-\frac{1}{4 d_{i}} \sum_{j, k=1}^{\ell}[i j k] \frac{2 x_{k}^{2}-x_{i}^{2}}{x_{i} x_{j} x_{k}}=\lambda \quad, \quad 1 \leq i \leq \ell
$$

where $b_{i}$ and $[i j k]$ are structure constants of $G / H$ and $d_{i}=\operatorname{dim} \mathfrak{m}_{i}$ (cf. [WZ2], [PS]). The problem is then to show that these equations have only finitely many (positive) real solutions.

In the general case, if some of the summands $\mathfrak{m}_{i}$ are equivalent, the scalar curvature function $S$ and the Einstein equations become more complicated. For instance, if there are several trivial summands, then examples are known, especially when $H=\{e\}$, where there exists a positivedimensional orbit of an Einstein metric under the gauge group $N(H) / H$, see e.g. Example 6.9. For such homogeneous spaces, the Einstein equations are not generic, i.e., they do not admit at most finitely many complex solutions.

Another important consequence of Theorem A is that it can be used to carry out LyusternikSchnirelmann theory type arguments for the existence of critical points. We emphasize though that $S$ is in general not a Morse-Bott function, since even the symmetric metric on an irreducible symmetric space can be degenerate. To carry out Lyusternik-Schnirelmann theory, we study directions in $\mathcal{M}_{1}^{G}$ where the scalar curvature goes to positive infinity. As was already observed in [WZ2], this is closely related to the existence of compact subgroups $K$ with $H \subset K \subset G$ such that $K / H$ is not a torus and $\operatorname{dim} K / H>0$. In this case, by the well-known formula [Bes, 9.71], the scalar curvature goes to $+\infty$ if one shrinks the fibers in the Riemannian submersion $G / H \rightarrow G / K$ to a point. Such subgroups $K$ correspond to subalgebras $\mathfrak{k}$ with $\mathfrak{h} \subset \mathfrak{k} \subset \mathfrak{g}$ which are $A d(H)$-invariant. We therefore associate to $G / H$ a graph whose vertices are (connected components of) $A d(H)$-invariant intermediate subalgebras $\mathfrak{k}$ and whose edges correspond to inclusions between two subalgebras. A component of the graph is called toral if all subalgebras in this component are such that the identity component of $K / H$ is abelian.

Our second main result is:

Theorem B. (Graph Theorem) Let $G / H$ be a compact homogeneous space. If the graph of $G / H$ has at least two non-toral components, then $G / H$ admits a homogeneous Einstein metric.

This theorem can be viewed as another step towards a general understanding of the existence and non-existence of homogeneous Einstein metrics, and was suggested by the last two authors 15 years ago.

In order to prove the Graph Theorem we will show that the number of components of high energy levels of $S$ is bounded from below by the number of non-toral components of the graph. We then connect a metric in such a component, corresponding to a metric on $G / H$ scaled down in the direction of a subgroup $K$, to a normal homogeneous background metric by a path of positive scalar curvature metrics. Hence two non-toral components give rise to a path of positive scalar curvature metrics which cannot be deformed to infinity by the gradient flow of $S$. Using Theorem A and a standard mountain pass lemma we obtain a critical point of coindex 1 (or 0 in the degenerate case), where the coindex of $g$ is the number of positive eigenvalues of the Hessian of $S$ at $g$. It is natural to ask how one can find critical points of higher coindex. One approach has recently been given by $\mathrm{C}$. Böhm in [Bö2], where he associates to each homogeneous space 
a simplicial complex, and shows that a nonvanishing homology group of this complex gives rise to a critical point of higher coindex.

One of the simplest examples which illustrates the proof of the Graph Theorem is the coadjoint orbit $U(p+q+r) / U(p) U(q) U(r)$, where the only (connected) intermediate subgroups are $K=$ $U(p+q) U(r), U(r+p) U(q)$ and $U(q+r) U(p)$. Hence the graph consists of three isolated vertices. It is well-known that these spaces admit homogeneous Kähler-Einstein metrics (cf. [Bes, 8.111]). In addition, all $U(p+q+r)$-invariant Einstein metrics have been explicitly determined in [Ar] and [Ki]. The pictures in Figure 1 of the graph and level curves of $S$ exhibit the three KählerEinstein metrics as saddle points and the three subgroup directions where $S \rightarrow+\infty$. A fourth critical point (non-Kähler) which is a local minimum appears in the middle.
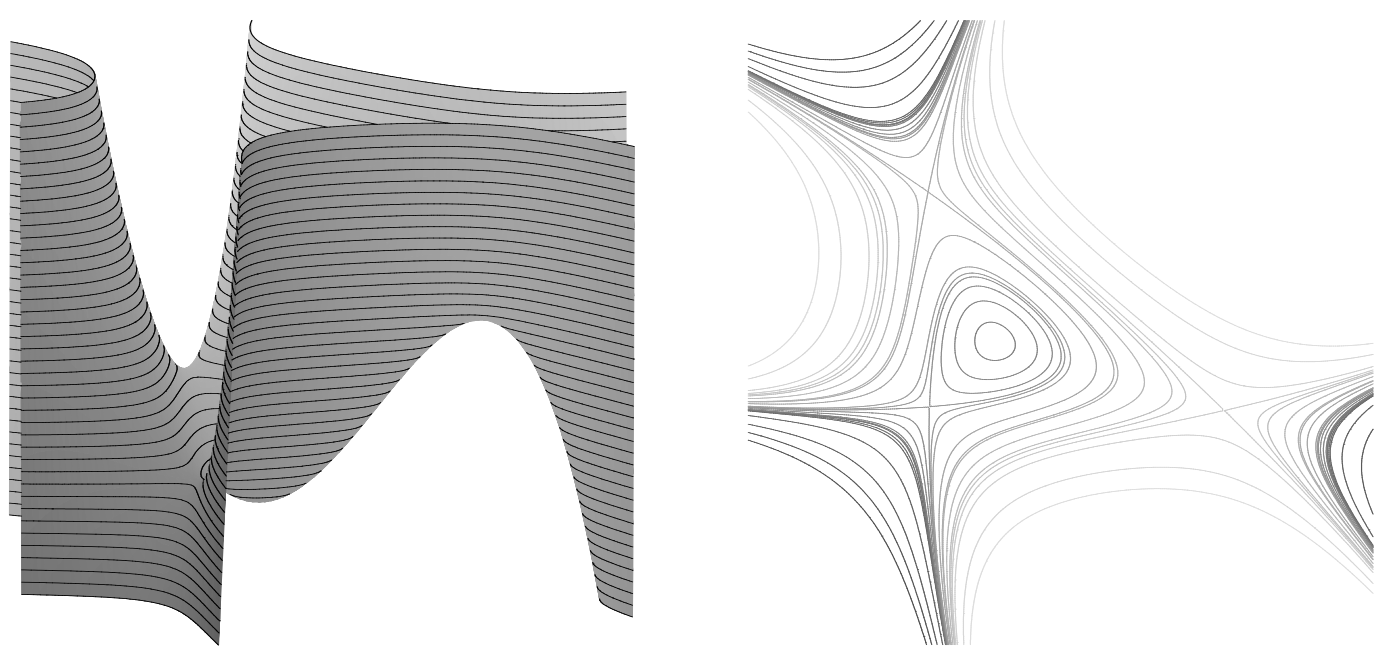

Figure 1. Einstein metrics on $U(9) / U(2) U(3) U(4)$.

Some non-trivial examples (where explicit solutions seem impossible) are $(G \times G) / \Delta H$ where $G$ is a simple Lie group and $H$ a maximal subgroup of $G$, or $G / S^{1}$ such that $S^{1} \subset S U(2) \subset G$ and $S U(2)$ is maximal in $G$. In $\S 6$ we will provide many other examples and further pictures which illustrate the possible local and global behavior of $S$. A Maple Demo that includes these examples can be found at the web site http://www.math.upenn.edu/ wziller/research.

Finally, let us turn to the Yamabe invariant $y(M)$ of a closed manifold $M$. This is the supremum of the scalar curvatures of all Yamabe metrics on $M$ (cf. [Ko], [Sc2]) where by a Yamabe metric we mean one with the smallest scalar curvature among all unit volume metrics in a conformal class. These metrics exist as a result of [Ya], [Tr], [Au1], [Sc1], (cf. [LP]). Since Einstein metrics of volume 1 are Yamabe metrics, one may ask whether a given Einstein metric on $M$ realizes $y(M)$. This never happens for Einstein metrics of negative scalar curvature on closed simply connected manifolds of dimension $>4$ because by [Pe] all such manifolds have non-negative Yamabe invariant. In $\S 5$ we derive the following criterion for the non-realization of the Yamabe invariant.

Proposition. Let $\left(M, g_{\infty}\right)$ be a closed unit volume Einstein manifold of dimension $n \geq 3$ not conformally equivalent to the round sphere. Suppose that there exists a sequence $g_{i}$ of unit volume metrics with constant scalar curvature $S_{g_{i}}>S_{g_{\infty}}$ converging to $g_{\infty}$ in the $C^{3}$ topology. Then $g_{\infty}$ cannot realize the Yamabe invariant of $M$. 
This result implies that the Einstein metrics obtained from the Graph Theorem, e.g., the Kähler-Einstein metrics in Figure 1, do not realize the Yamabe invariant. Of course, in general these homogeneous Einstein metrics may not be a "best" homogeneous Einstein metric on $M$, i.e., ones with largest scalar curvature. But we will also show that sometimes even the best homogeneous Einstein metric does not realize the Yamabe invariant, e.g., the symmetric metrics of the irreducible symmetric spaces $S p(n) / U(n)$ and $S U(2 n) / S p(n)$.

Although the Yamabe maximin procedure may not in general produce an Einstein metric on $M$, we can nevertheless make the following comparison. If we fix a transitive $G$-action on $M$, one may consider the $G$-invariant analog of the Yamabe maximin procedure. A non-trivial conformal deformation of a $G$-invariant unit volume metric can never be $G$-invariant. So the maximin procedure corresponds to maximizing the scalar curvature function over the space of $G$-invariant metrics of unit volume. This is precisely the situation analyzed in [WZ2] and Theorem 3.2. What we have attempted in this paper then is the $G$-homogeneous analog of the next level of maximin variation schemes which produce critical points of small coindex.

Our paper contains six sections. In the first section we prove Theorem A and discuss the consequences for the moduli space of homogeneous Einstein metrics. In $\S 2$ we examine 0-PalaisSmale sequences. The Graph Theorem is proved in $\S 3$, and general properties of the graph are examined in $\S 4$. In $\S 5$ we discuss the relationship of our results to the Yamabe invariant, and in $\S 6$ we give some examples illustrating various aspects of our general theory.

\section{A Palais-Smale Condition for Homogeneous Metrics}

Let $M$ be an $n$-dimensional closed connected smooth manifold. Let $\mathcal{M}$ denote the space of all smooth Riemannian metrics on $M$. This space has a natural Riemannian metric, the $L^{2}$ metric, which is given by

$$
\|h\|_{g}^{2}=\int_{M} g(h, h) d \mu_{g},
$$

where $h$ is a symmetric 2-tensor (considered as a tangent vector at the metric $g$ ), $d \mu_{g}$ is the volume element of $g$, and we have extended $g$ in the usual way to inner products on all tensor bundles over $M$. The Hilbert action is the functional which associates to a metric $g$ the integral of its scalar curvature $S_{g}$ :

$$
\mathbf{S}(g)=\int_{M} S_{g} d \mu_{g}
$$

From now on we will restrict the Hilbert action to the subspace $\mathcal{M}_{1} \subset \mathcal{M}$ of unit volume metrics. It follows from the well-known first variation formula

$$
d \mathbf{S}_{g}(h)=\int_{M} g\left(\frac{S_{g}}{n}-\operatorname{Ric}(g), h\right) d \mu_{g}
$$

that the $L^{2}$ gradient of $\mathbf{S}$ on $\mathcal{M}_{1}$ is the negative of the traceless Ricci tensor of $g$, which we shall denote by $\operatorname{Ric}^{0}(g)$.

Suppose next that $M$ is in addition homogeneous under the action of some compact Lie group $G$, i.e., $M=G / H$. If we choose a base point $x_{0} \in M$, then one obtains a specific equivariant diffeomorphism $G / H \approx M$ by sending $g H$ to $g \cdot x_{0}$. Such identifications used below will not be mentioned explicitly. Note that there could be infinitely many inequivalent transitive actions of $G$ on $M$ (see [WZ3]), and that two transitive $G$-actions on $M$ are equivalent iff the isotropy groups at the base point $x_{0}$ are conjugate.

Let $\left\{g_{i}\right\}$ be a sequence of unit volume $G$-homogeneous metrics on $M$, not necessarily for the same transitive action of $G$. By homogeneity, $\mathbf{S}\left(g_{i}\right)$ equals the scalar curvature $S_{i}:=S_{g_{i}}$ of $g_{i}$, and the sequence of $L^{2}$ norms

$$
\left\|\nabla \mathbf{S}_{g_{i}}\right\|_{g_{i}}=\left\|\operatorname{Ric}^{0}\left(g_{i}\right)\right\|_{g_{i}}
$$


is just the sequence of pointwise norms of the traceless Ricci tensors with respect to $g_{i}$. Now suppose that $\left|S_{i}\right|$ are bounded and $\left\|\operatorname{Ric}^{0}\left(g_{i}\right)\right\|_{g_{i}}$ converge to 0 . After passing to a subsequence, we may assume that $S_{i}$ converge to some $\lambda \geq 0$. For if $\lambda<0$, then we would have homogeneous metrics with negative Ricci tensor on $M$, which would contradict a theorem of Bochner.

We shall consider the case in which $\lambda>0$ in this section and the case $\lambda=0$ in the next. The main result of this section is:

THEOREm 1.1. Let $G$ be a compact Lie group and let $\left\{g_{i}\right\}$ be a sequence of unit volume $G$ homogeneous metrics on a closed connected pointed n-manifold $\left(M \approx G / H_{i}, x_{0}\right)$, where $H_{i}$ is the isotropy group of the base point $x_{0}$ for the $i$ th transitive $G$-action $\Psi_{i}$. Suppose that the scalar curvatures $S_{i}$ converge to $\lambda>0$ and that $\left\|\operatorname{Ric}^{0}\left(g_{i}\right)\right\|_{g_{i}} \rightarrow 0$. Then there exists a subsequence which converges in the $C^{\infty}$ topology to a $G$-homogeneous Einstein metric $g_{\infty}$ on $M$ with positive scalar curvature. Furthermore, if the transitive $G$-actions $\Psi_{i}$ on $M$ are identical, then $g_{\infty}$ is homogeneous with respect to the same $G$-action.

Proof. The assumptions on the sequence $\left\{g_{i}\right\}$ imply that, after passing to a subsequence, there is a uniform positive lower (and of course upper) bound on the Ricci curvatures of $\left\{g_{i}\right\}$. By Myers' theorem, we obtain a uniform upper diameter bound, and the Bishop-Gromov volume comparison theorem [Pe2, p. 247] shows that there is a uniform positive lower bound for the volumes of unit metric balls in $\left(M, g_{i}\right)$. We are thus in a position to apply Theorem 7.2 in [CC] of Cheeger and Colding.

This result gives a subsequence of $\left(M \approx G / H_{i}, x_{0}, g_{i}\right)$ which converges in the pointed Gromov-Hausdorff topology to a pointed complete metric space $\left(Y, y_{\infty}, d_{\infty}\right)$ of the same Hausdorff dimension. (In the interest of making notation as simple as possible, we will not distinguish between the original sequence and its subsequences in the following.)

This theorem asserts further that there is an open submanifold $R \subset Y$ and, on it, a Riemannian metric $g_{\infty}$, compatible with $d_{\infty}$, of class $C^{1, \alpha}$ (with $0<\alpha<1$ ) such that at points of $R$ the convergence $\left(M \approx G / H_{i}, x_{0}, g_{i}\right) \rightarrow\left(Y, y_{\infty}, d_{\infty}\right)$ takes place in the $C^{1, \alpha}$ topology. Using the transitive actions of $G$, the smoothness of $G / H_{i}$, and the definition of pointed Gromov-Hausdorff convergence (in terms of the convergence of metric balls in the pointed Gromov-Hausdorff topo$\operatorname{logy}$ ), it follows that $R=Y$. Therefore, for every $r>0$, there exists a domain $\Omega_{r} \subset Y$ containing the metric ball $B_{\infty}\left(y_{\infty}, r\right)$ and for large $i$, there are embeddings

$$
\phi_{i}^{(r)}:\left(\Omega_{r}, y_{\infty}\right) \hookrightarrow\left(M \approx G / H_{i}, x_{\infty}\right)
$$

such that $B_{i}\left(x_{0}, r\right) \subset \phi_{i}^{(r)}\left(\Omega_{r}\right)$ and on $\Omega_{r}$ the metrics $\left(\phi_{i}^{(r)}\right)^{*} g_{i}$ converge in the $C^{1, \alpha}$ topology to $g_{\infty}$ (as tensor fields). Finally note that, since the Ricci curvatures of $g_{i}$ converge to $\lambda / n$, the same is true for the pulled-back metrics $\left(\phi_{i}^{(r)}\right)^{*} g_{i}$, and the $C^{1, \alpha}$ convergence implies that $g_{\infty}$ is an Einstein metric in the $C^{1, \alpha}$ sense. By elliptic regularity, $g_{\infty}$ is a smooth Einstein metric with Einstein constant $\lambda / n$ (see p. 288 of [Pe2] for further details).

Therefore, we obtain a subsequence of $\left(M \approx G / H_{i}, x_{0}, g_{i}\right)$ which converges in the $C^{1, \alpha}$ topology to a smooth compact Einstein manifold $\left(Y, y_{\infty}, g_{\infty}\right)$. In particular, (for large $i$ ) there are base-point preserving diffeomorphisms

$$
\phi_{i}:\left(Y, y_{\infty}\right) \longrightarrow\left(M \approx G / H_{i}, x_{0}\right)
$$

so that the gauge-transformed metrics $\phi_{i}^{*} g_{i}$ converge to $g_{\infty}$ in $C^{1, \alpha}$. Furthermore, by the corollary on p. 66 of $[\mathrm{Gr}]$, the Einstein metric $g_{\infty}$ is homogeneous. We note that the arguments up to this point allow us to replace $G$ by a sequence of transitive compact Lie groups $G_{i}$ with isotropy group $H_{i}$. 
The remainder of the proof concerns the subconvergence of the transitive $G$-actions, the isometries of $g_{\infty}$, and the subconvergence in the $C^{\infty}$ topology of the sequence $g_{i}$ itself.

Using the diffeomorphisms $\phi_{i}$ above, we obtain transitive actions $\Theta_{i}$ on $Y$ by letting $\Theta_{i}(g, y):=$ $\phi_{i}^{-1}\left(\Psi_{i}\left(g, \phi_{i}(y)\right)\right)$. Note that the isotropy group of $y_{\infty}$ for $\Theta_{i}$ is $H_{i}$ and $\Theta_{i}$ is an isometric $G-$ action for the metric $\hat{g}_{i}:=\phi_{i}^{*} g_{i}$. Let us now consider the sequence of Riemannian $G$-manifolds $\left(Y, \Theta_{i}, y_{\infty}, \hat{g}_{i}\right)$, which has a lower Ricci and an upper diameter bound. By Theorem 6.9 in [Fu], which is an equivariant version of the Gromov compactness theorem, there is a subsequence which converges in the equivariant Gromov-Hausdorff topology to $\left(Y, \Theta_{\infty}, y_{\infty}, g_{\infty}\right)$ with an isometric $G$-action $\Theta_{\infty}$.

Claim 1: There exists an isometry $a_{\infty}$ of $\left(Y, g_{\infty}\right)$ such that the $G$-actions $\Theta_{i}$ subconverge uniformly to the transitive $G$-action $\Theta^{*}$ defined by $\Theta^{*}(g, y)=a_{\infty}^{-1}\left(\Theta_{\infty}\left(g, a_{\infty}(y)\right)\right)$.

Claim 2: Let $H_{\infty}$ denote the isotropy group of the $G$-action $\Theta^{*}$ at $y_{\infty}$. For sufficiently large $i$, there exist elements $u_{i} \in G$ such that $u_{i}$ converge to 1 and $u_{i} H_{i} u_{i}^{-1}=H_{\infty}$.

We will postpone the proofs of claims 1 and 2 to the end and continue with the proof of the theorem. Now claim 2 implies that the elements $u_{i}$ induce $G$-equivariant diffeomorphisms $R_{i}: G / H_{\infty} \rightarrow G / H_{i} \approx Y$ given by $R_{i}\left(g H_{\infty}\right)=g u_{i} H_{i}$. Thus a subsequence of the actions $\Psi_{i}$ eventually become all equivalent to $\Theta^{*}$. Since $u_{i} \rightarrow 1$, the metrics $R_{i}^{*} \hat{g}_{i}=\left(\phi_{i} \circ R_{i}\right)^{*} g_{i}$ converge in $C^{1, \alpha}$ to $g_{\infty}$. But $R_{i}^{*} \hat{g}_{i}$ and $g_{\infty}$ are invariant metrics with respect to the fixed transitive $G$-action $\Theta^{*}$. So the convergence of $R_{i}^{*} \hat{g}_{i}$ to $g_{\infty}$ takes place in $C^{\infty}$. Finally, observe that $\phi_{i} \circ R_{i}$ is a $G$-equivariant diffeomorphism $G / H_{\infty} \rightarrow G / H_{i} \approx M$, and so it is given by right translation by some element $b_{i}^{-1} \in G$. Since $G$ is compact, we may assume that $b_{i}$ converges to $b_{\infty}$ lying in the normalizer of $H_{\infty}$ in $G$. So $g_{i}$ subconverges in the $C^{\infty}$ topology to the metric $R_{b_{\infty}}^{*} g_{\infty}$, which is $\Theta^{*}$-invariant.

Of course, if the $G$-actions $\Psi_{i}$ are the same, i.e., the homogeneous structure on $M$ is fixed, then $H_{i}=H_{\infty}$ for all $i$ and the metric $R_{b_{\infty}}^{*} g_{\infty}$ is homogeneous with respect to the fixed action. It remains to prove the above two claims.

Proof of Claim 1. Let $\epsilon_{i}=2^{-i}$ and $\hat{d}_{i}$ denote the distance function underlying the metric $\hat{g}_{i}$. Since $\left(Y, \Theta_{i}, \hat{d}_{i}\right)$ converges in the equivariant Gromov-Hausdorff topology to $\left(Y, \Theta_{\infty}, d_{\infty}\right)$, there exist equivariant $\epsilon_{i}$-approximations $a_{i}:\left(Y, \Theta_{i}, \hat{d}_{i}\right) \rightarrow\left(Y, \Theta_{\infty}, d_{\infty}\right)$ (not necessarily continuous) such that the $\epsilon_{i}$-neighborhood of $a_{i}(Y)$ in $Y$ is $Y$, and for arbitrary elements $x, y \in Y, g \in G$ we have

$$
\begin{aligned}
\left|\hat{d}_{i}(x, y)-d_{\infty}\left(a_{i}(x), a_{i}(y)\right)\right| & <\epsilon_{i} \\
d_{\infty}\left(a_{i}\left(\Theta_{i}(g, x)\right), \Theta_{\infty}\left(g, a_{i}(x)\right)\right) & <\epsilon_{i}
\end{aligned}
$$

We show next that the family $\left\{a_{i}\right\}$ is equicontinuous. To see this, let $\epsilon>0$ be given and set $\delta:=\epsilon / 3$. Since $\hat{g}_{i}$ converges to $g_{\infty}$ in $C^{0}$, for sufficiently large $i$ we may assume that $\epsilon_{i}<\epsilon / 3$ and for all $x, y \in Y$ we have $\left|\hat{d}_{i}(x, y)-d_{\infty}(x, y)\right|<\epsilon / 3$. It follows that if $d_{\infty}(x, y)<\delta$ and $i$ is sufficiently large, we have

$$
\left|d_{\infty}\left(a_{i}(x), a_{i}(y)\right)-d_{\infty}(x, y)\right| \leq\left|d_{\infty}\left(a_{i}(x), a_{i}(y)\right)-\hat{d}_{i}(x, y)\right|+\left|\hat{d}_{i}(x, y)-d_{\infty}(x, y)\right|<\frac{2}{3} \epsilon
$$

using (1.2). This implies that $d_{\infty}\left(a_{i}(x), a_{i}(y)\right)<d_{\infty}(x, y)+\frac{2}{3} \epsilon<\epsilon$. Then the Arzela-Ascoli argument (see p. 177-178 of [Roy]) gives a subsequence of $\left\{a_{i}\right\}$ which converges uniformly on $Y$ to $a_{\infty}$, which must be an isometry of $d_{\infty}$ and hence a diffeomorphism of $Y$.

We can now finish the proof of claim 1. Let $\rho$ be any distance function on $G$. Given $\epsilon>0$, since $\Theta_{\infty}: G \times Y \rightarrow Y$ is uniformly continuous, we can find $0<\delta<\frac{\epsilon}{3}$ such that whenever

$$
\rho\left(g_{1}, g_{2}\right)+d_{\infty}\left(y_{1}, y_{2}\right)<\delta
$$


we have

$$
d_{\infty}\left(\Theta_{\infty}\left(g_{1}, y_{1}\right), \Theta_{\infty}\left(g_{2}, y_{2}\right)\right)<\epsilon / 3 .
$$

Now the uniform convergence of the maps $\left\{a_{i}\right\}$ to $a_{\infty}$ can be used to choose $i_{0}$ so that for all $i>i_{0}$,

$$
d_{\infty}\left(a_{\infty}(y), a_{i}(y)\right)<\delta \quad \text { for all } y \in Y .
$$

For all such $i$, we then have

$$
\begin{aligned}
d_{\infty}\left(a_{\infty}^{-1}\left(\Theta_{\infty}\left(g, a_{\infty}(y)\right)\right), \Theta_{i}(g, y)\right) \leq & d_{\infty}\left(a_{\infty}^{-1}\left(\Theta_{\infty}\left(g, a_{\infty}(y)\right)\right), a_{\infty}^{-1}\left(\Theta_{\infty}\left(g, a_{i}(y)\right)\right)\right) \\
& +d_{\infty}\left(a_{\infty}^{-1}\left(\Theta_{\infty}\left(g, a_{i}(y)\right)\right),\left(a_{\infty}^{-1} \circ a_{i}\right)\left(\Theta_{i}(g, y)\right)\right) \\
& +d_{\infty}\left(\left(a_{\infty}^{-1} \circ a_{i}\right)\left(\Theta_{i}(g, y)\right), \Theta_{i}(g, y)\right) .
\end{aligned}
$$

The first term on the right-hand side is smaller than $\epsilon / 3$ using (1.4) and the uniform continuity of $\Theta_{\infty}$. The second term is smaller than $\epsilon / 3$ by (1.3). Finally, the third term is smaller than $\epsilon / 3$ by the uniform convergence of $a_{\infty}^{-1} \circ a_{i}$ to the identity map.

Proof of Claim 2. Let $\eta_{i}: G \rightarrow Y$ and $\eta_{\infty}: G \rightarrow Y$ denote the maps defined by taking $g \in G$ to $\Theta_{i}\left(g, y_{\infty}\right)$ and $\Theta^{*}\left(g, y_{\infty}\right)$, respectively. Choose a neighborhood $U$ of $1 \in G$. A classical result of Montgomery and Zippin (see the Theorem, p. 216 in [MZ]) asserts that there is an open set $\mathcal{O} \supset H_{\infty}$ such that any compact subgroup of $G$ lying in $\mathcal{O}$ is conjugate to a subset of $H_{\infty}$ by some element $u \in U$. Since $\eta_{\infty}$ is an orbit projection and $H_{\infty}$ is compact, we may assume that $\mathcal{O}$ contains the inverse image under $\eta_{\infty}$ of some $d_{\infty}$-metric ball $B\left(y_{\infty}, \epsilon\right) \subset Y$. By claim $1, \eta_{i}$ subconverges uniformly to $\eta_{\infty}$. So for large enough $i$ and all $h_{i} \in H_{i}$, we have

$$
d_{\infty}\left(y_{\infty}, \eta_{\infty}\left(h_{i}\right)\right)=d_{\infty}\left(\eta_{i}\left(h_{i}\right), \eta_{\infty}\left(h_{i}\right)\right)<\epsilon .
$$

This shows that $H_{i} \subset \mathcal{O}$, and hence there exists $u_{i} \in U$ with $u_{i} \rightarrow 1$ such that $u_{i} H_{i} u_{i}^{-1} \subset H_{\infty}$. But the volume of $\hat{g}_{i}$ converges to the volume of $g_{\infty}$. So we actually have $u_{i} H_{i} u_{i}^{-1}=H_{\infty}$.

This completes the proof of claim 2 and also that of Theorem 1.1.

We will next deduce several corollaries of Theorem 1.1. First, we consider the situation in which the Lie group $G$ is not fixed.

Given a compact connected homogeneous $n$-manifold $M$ with $n>0$, there can be infinitely many abstract isomorphism classes of compact Lie groups which act effectively and transitively on $M$. A simple example is the following. Let $G^{0}$ be a simple compact connected Lie group and $\Gamma$ be any finite subgroup. Then $\left(G^{0} \times \Gamma\right) / \Delta \Gamma$, where $\Delta \Gamma$ is embedded diagonally, is diffeomorphic to $G^{0}$ and effective provided that $\Gamma \cap Z\left(G^{0}\right)=\{1\}$. In particular, there are infinitely many cyclic subgroups in a compact circle subgroup in $G^{0}$ which have this property. By contrast, for compact connected Lie groups acting almost effectively and transitively on $M$, their abstract isomorphism classes are finite in number. To see this, note first that the dimensions of the groups are bounded between $n$ and $\frac{1}{2} n(n+1)$. Such a group is of the form $\tilde{G} / N$, where $\tilde{G}=T^{k} \times G_{1} \times \cdots \times G_{\ell}$, and $G_{i}$ are simply connected, compact, simple Lie groups. The dimension restrictions limit the choices of $k$ and $G_{i}$ to finitely many. There can also be only finitely many quotients of $\tilde{G}$ by finite central subgroups $N$ because there are finitely many possibilities for the centers of $G_{i}$, and because the quotient of a torus by a finite subgroup is again a torus of the same rank. Finally let us note that since $M$ is connected, the connected component $G_{i}^{0}$ of $G_{i}$ containing the identity still acts transitively on $M$; that is, $G_{i} / H_{i}=G_{i}^{0} /\left(G_{i}^{0} \cap H_{i}\right)$ as manifolds. We also may assume that $M=G_{i}^{0} /\left(G_{i}^{0} \cap H_{i}\right)$ is presented as an almost effective homogeneous space. The following corollary is now an immediate consequence of Theorem 1.1.

Corollary 1.5. Let $\left\{g_{i}\right\}$ be a sequence of unit volume $G_{i}$-homogeneous metrics on the closed connected almost effective homogeneous spaces $M \approx G_{i} / H_{i}$. Suppose that the scalar curvatures 
$S_{i}$ converge to $\lambda>0$ and that $\left\|\operatorname{Ric}^{0}\left(g_{i}\right)\right\|_{g_{i}} \rightarrow 0$. Then there exists a compact connected Lie group $G$ and a subsequence of $\left\{g_{i}\right\}$ which converges in the $C^{\infty}$ topology to a G-homogeneous Einstein metric $g_{\infty}$ on a compact homogeneous space $M^{n}=G / H$ with positive scalar curvature such that $G=G_{i}^{0}$ for all $i \in \mathbb{N}$.

Next, we need to establish some notation. Suppose that $G$ is a compact Lie group acting transitively on our manifold $M$ with base point $x_{0}$ and isotropy group $H$. Let $\mathcal{M}_{1}^{G}$ denote the space of $G$-invariant metrics on $G / H$ with volume 1 , and $\mathcal{E}(G / H)$ denote the subset of $G$-invariant Einstein metrics in $\mathcal{M}_{1}^{G}$ (endowed with the relative topology).

We will fix a bi-invariant background metric $Q$ on $G$ once and for all. This gives us a $Q$ orthogonal decomposition

$$
\mathfrak{g}=\mathfrak{h} \oplus \mathfrak{m}
$$

of the Lie algebra $\mathfrak{g}$ of $G$, which is $A d(H)$-invariant. The $A d(H)$-representation on $\mathfrak{m}$ is known as the isotropy representation of $G / H$. Using the above decomposition, we may identify the tangent space of $G / H$ at the coset $(1 H)$ with $\mathfrak{m}$, and $G$-invariant metrics on $G / H$ with $A d(H)$ invariant inner products on $\mathfrak{m}$. In particular, $Q$ induces a normal homogeneous metric on $G / H$ which we may assume to have volume 1 (after rescaling).

Now given $g \in \mathcal{M}_{1}^{G}$, via the above identifications, we may write, for $X, Y \in \mathfrak{m}, g(X, Y)=$ $Q\left(\alpha_{g}(X), Y\right)$, where $\alpha_{g}$ is a determinant 1, positive-definite element of $S^{2}(\mathfrak{m})^{A d(H)}$, the space of $A d(H)$-equivariant, $Q$-self-adjoint endomorphisms of $\mathfrak{m}$. More globally, $\alpha_{g}$ can be regarded as an $A d(H)$-equivariant element of the symmetric space $S L(\mathfrak{m}) / S O(\mathfrak{m}, Q)$. As explained in [Es], this association gives an isometric embedding of $\mathcal{M}_{1}^{G}$, equipped with the restriction of the $L^{2}$ metric of $\mathcal{M}_{1}$, into $S L(\mathfrak{m}) / S O(\mathfrak{m}, Q)$, equipped with the symmetric metric, as a symmetric subspace.

The above description shows that $\mathcal{M}_{1}^{G}$ has trivial topology, while as a Riemannian manifold (with the $L^{2}$ metric), it is complete. But it need not be de Rham irreducible, as it is in the case of left-invariant metrics on a compact Lie group. For instance, if the isotropy representation of $G / H$ consists of pairwise inequivalent irreducible subrepresentations, then $\left(\mathcal{M}_{1}^{G}, L^{2}\right)$ is flat with dimension equal to the number of irreducible summands.

Proposition 1.6. The space $\mathcal{E}(G / H)$ is a semialgebraic set and hence has finitely many topological components. Locally, it has a stratification into real algebraic submanifolds. In particular, it is locally path-connected, so that path components and topological components coincide.

Proof. We view $\mathcal{E}(G / H)$ as a subset of $S^{2}(\mathfrak{m})^{A d(H)} \times \mathbb{R}$, where a $G$-invariant Einstein metric $g$ with (constant) scalar curvature $S$ corresponds to the point $\left(\alpha_{g}, S\right)$. If we fix an orthonormal basis of the metric $\left.Q\right|_{\mathfrak{m}}$, then the Einstein condition for $g$ can be written down by polarising formula (7.38) on p. 185 of [Bes]:

$$
R_{a b}=-\frac{1}{2} B_{a b}-\frac{1}{2} \sum_{i, j, k, l} C_{a i}^{k} C_{b j}^{l} g_{k l} g^{i j}+\frac{1}{4} \sum_{i, j, k, l, p, q} C_{i k}^{p} C_{j l}^{q} g_{p a} g_{q b} g^{i j} g^{k l},
$$

where $B_{a b}$ and $C_{i j}^{k}$ are constants and $g^{i j}$ are the components of the inverse of $\left(g_{i j}\right)$. Hence each equation in the Einstein system is of the form $P_{i} / \operatorname{det}\left(\alpha_{g}\right)^{2}=0$, where $P_{i}$ is a polynomial in $S$ and the entries of $\alpha_{g}$. Such an element in $\mathcal{E}(G / H)$ is specified by the equations $P_{i}\left(\alpha_{g}, S\right)=0$, $\operatorname{det}\left(\alpha_{g}\right)=1$, and a finite number of additional inequalities $F_{j}\left(\alpha_{g}\right)>0$ expressing the positivedefiniteness of $\alpha_{g}$. Hence $\mathcal{E}(G / H)$ is a semialgebraic set. The properties we listed for it are classical theorems of $\mathrm{H}$. Whitney. (See $[\mathrm{Wh}]$, or $[\mathrm{BCR}]$ for a more modern reference.)

Now suppose we have a connected homogeneous space $G / H$ with $G$ compact as above. If $\mathcal{E}(G / H)$ is non-empty and there is an element with zero scalar curvature, then $[\mathrm{AK}]$ implies that 
the Einstein metric is flat. Hence $G / H$ is a torus, and all other $G$-invariant Einstein metrics are also flat. If $G / H$ is not a torus, then all $G$-invariant Einstein metrics have positive scalar curvature.

THEOREM 1.7. Let $G$ be a compact Lie group and $G / H$ be a connected homogeneous space with finite fundamental group. Then each component of $\mathcal{E}(G / H)$ is compact, and the set of Einstein constants of metrics in $\mathcal{E}(G / H)$ is finite.

Proof. Recall first that Einstein constants along a smooth path of Einstein metrics with unit volume must remain unchanged (see [B], p. 289). The local stratification of $\mathcal{E}(G / H)$ by algebraic submanifolds provided by Proposition 1.6 means that two points in the same component can be joined by a piecewise real analytic path. Hence the Einstein constants of all metrics in the same component are the same, and there can only be finitely many Einstein constants. Compactness in $\mathcal{E}(G / H)$ is equivalent to sequential compactness, which follows immediately from Theorem 1.1 .

Finally we turn back to the situation in which the homogeneous structure is not fixed. Let $\varepsilon_{h}(M)$ denote the space of unit volume homogeneous Einstein metrics on $M$ with topology induced from the $C^{\infty}$ topology of $\mathcal{M}_{1}$. For any real number $\lambda$, let $\bar{\varepsilon}_{h}(M)_{\lambda}$ denote the subspace of $\varepsilon_{h}(M) / \operatorname{Diff}(M)$ consisting of Riemannian structures with scalar curvature $\geq \lambda$.

TheOREM 1.8. Let $M$ be a homogeneous compact connected manifold. For each positive number $\lambda$, the space $\overline{\mathcal{E}}_{h}(M)_{\lambda}$ has finitely many components and each component is compact. In particular, there is a maximum value for the Einstein constants of the unit volume homogeneous Einstein metrics on $M$.

Proof. Given a sequence lying in a component of $\bar{\varepsilon}_{h}(M)_{\lambda}$, by lifting we obtain a sequence of homogeneous metrics whose scalar curvatures must be bounded from above as well by the Bishop volume comparison theorem. Corollary 1.5 implies now that there is a convergent subsequence and hence compactness is proved.

If $\bar{\varepsilon}_{h}(M)_{\lambda}$ had infinitely many components, we could form a sequence of $G$-homogeneous Einstein metrics coming from distinct components. By the proof of Theorem 1.1, after applying suitable diffeomorphisms, we would have a convergent subsequence of $G$-homogeneous Einstein metrics for a fixed homogeneous structure $G / H$. By Theorem 1.7, there is a further subsequence which lies in one component of $\mathcal{E}(G / H)$. Observe that the topology of $\mathcal{E}(G / H)$ is compatible with the $C^{\infty}$ topology of $\mathcal{E}_{h}(M)$. Hence the image of this subsequence in $\overline{\mathcal{E}}_{h}(M)_{\lambda}$ would also lie in a single component, which is a contradiction. The same argument shows that there can only be finitely many different Einstein constants in this set.

Since in the proof of Theorem 1.1 the manifold does not have to be fixed, we also have the following consequence:

Corollary 1.9. For fixed $n$ and for every $\epsilon>0$ there exist at most finitely many diffeomorphism types of n-dimensional compact connected homogeneous spaces which admit homogeneous Einstein metrics with scalar curvature bigger or equal than $\epsilon$.

\section{0-Palais-Smale Sequences}

Let $M$ be a compact connected homogeneous $n$-manifold and $G_{i}$ be compact transitive groups on $M$ with isotropy group $H_{i}$ at some base point $x_{0}$. In this section we will consider sequences of homogeneous metrics $\left\{g_{i}\right\}$ such that $\left\|\operatorname{Ric}^{0}\left(g_{i}\right)\right\|_{g_{i}}$ and $S_{g_{i}}$ both converge to 0 . We shall refer 
to such sequences as 0-Palais-Smale sequences. Notice that a 0-Palais-Smale sequence cannot have a convergent subsequence if $M$ is not a torus.

0-Palais-Smale sequences can be constructed easily on homogeneous torus bundles as follows. Let $G$ be a compact Lie group, $H, K$ be closed subgroups such that $K \supset H$ and $K / H \approx T^{r}, r \geq$ 1. Denote by $g$ the normal homogeneous metric on $G / H$ induced by any bi-invariant metric on $G$ and by $g_{t}$ the 1-parameter family of homogeneous metrics formed from $g$ by scaling the metrics along $K / H$ by $t>0$. Finally let $\hat{g}_{t}=t^{-r / n} g_{t}$ denote the renormalised metrics with volume 1 . For $t_{k} \rightarrow 0$, we claim that $\hat{g}_{t_{k}}$ is a 0-Palais-Smale sequence. By an argument similar to that in the proof of Proposition 4.2 in [WZ3], and noting that the base metric and the torus bundle here (and hence its curvature form) are fixed, one sees that the sectional curvatures of $g_{t_{k}}$ are uniformly bounded. Hence the sectional curvature and thus the Ricci curvature and the scalar curvature of $\hat{g}_{t_{k}}$ converge to 0 . For the unnormalized sequence $g_{t}$ it follows that they collapse with bounded diameter to a smooth homogeneous metric on the lower dimensional manifold $G / K$, whereas the normalized sequence $\hat{g}_{t}$ collapses in the pointed Gromov-Hausdorff topology to a flat euclidean space of the same dimension.

The main result of this section is a converse of this observation.

THEOREM 2.1. Let $\left(M, x_{0}\right)$ be a pointed compact connected $n$-manifold with a sequence of transitive, compact Lie groups $G_{i}$. Let $H_{i}$ denoted the corresponding isotropy groups of $x_{0}$. Suppose that there exists a 0-Palais-Smale sequence $\left\{g_{i}\right\}$ of unit volume $G_{i}$-invariant metrics. Then there is a subsequence of $G_{i} / H_{i}$ such that each $G_{i}^{0} / H_{i}^{0}$ is a homogeneous torus bundle, i.e., for each $i$ there exists a subgroup $K_{i} \subset G_{i}^{0}$ containing $H_{i}^{0}$ with $K_{i}^{0} / H_{i}^{0}=T^{k}$.

This theorem is optimal if the isotropy groups $H_{i}$ are connected. For disconnected isotropy groups $H_{i}$ we conjecture that $G_{i} / H_{i}$ itself is a homogeneous torus bundle.

For the proof of this theorem, we will make use of the harmonic norm introduced by Petersen in [Pe1] and further discussed in [Pe2]. The harmonic norm is closely related to the harmonic radius introduced by M. Anderson in [An]. Specifically, we will make use of the $C^{1, \alpha}$ harmonic norm with scale $r$ for subsets $A$ in a Riemannian manifold, to be denoted by $\|A \subset M, g\|_{1, \alpha, r}^{h}$. When $A=M$ we will just write $\|M, g\|_{1, \alpha, r}^{h}$.

Proof of Theorem 2.1. Let $\left\{g_{i}\right\}$ be a 0-Palais-Smale sequence as above with unit volume. Consider the sequence of harmonic norms $\left\|M, g_{i}\right\|_{1, \alpha, r}^{h}$ where $0<\alpha<1$ and $r>0$ are fixed. There are two possibilities.

If the sequence is bounded above by some constant $C$, then by Corollary 10.5.1 in [Pe2], $\left(M, x_{0}, g_{i}\right)$ converges in the $C^{1, \beta}$ topology, for any fixed $0<\beta<\alpha$, to some complete Riemannian manifold $\left(M^{*}, x^{*}, g^{*}\right)$. By elliptic regularity as in the proof of Theorem 1.1, $g^{*}$ must be Einstein and hence Ricci-flat. But the volumes converge as well, and so $\operatorname{vol}\left(M^{*}, g^{*}\right)=1$. Furthermore, by the corollary on p. 66 of $[\mathrm{Gr}], M^{*}$ is homogeneous. So $M^{*}$ must be compact. By [AK], $g^{*}$ must be flat, and hence $M^{*}$ is a torus.

The second possibility is that, after passing to a subsequence, we have $\left\|M, g_{i}\right\|_{1, \alpha, r}^{h} \rightarrow+\infty$. Since $r$ was not specified in the above, we may assume that $r=1$ was chosen, and hence for large $i$ we have $\left\|M, g_{i}\right\|_{1, \alpha, 1}^{h}>1$. For a fixed Riemannian manifold and $\alpha>0$, the harmonic norm is a continuous function of the scale $r$ and tends to 0 as $r$ tends to 0 (cf. (2), p. 304 of $[\mathrm{Pe} 2])$. So we can find constants $0<r_{i}<1$ such that for large $\mathrm{i}$

$$
1=\left\|M, g_{i}\right\|_{1, \alpha, r_{i}}^{h}=\left\|M, r_{i}^{-2} g_{i}\right\|_{1, \alpha, 1}^{h} .
$$

Let $\hat{g}_{i}=r_{i}^{-2} g_{i}$. Then since $r_{i}<1,\left(M_{i}, \hat{g}_{i}\right)$ is still a 0 -Palais-Smale sequence, but with $\operatorname{vol}\left(\hat{g}_{i}\right) \rightarrow$ $+\infty$ whenever $r_{i} \rightarrow 0$. We apply next Corollary 10.5.4 in [Pe2] to conclude that $\left(M_{i}, x_{0}, \hat{g}_{i}\right)$ 
subconverges in the pointed $C^{1, \alpha}$ topology to a complete Riemannian manifold $\left(M^{*}, x^{*}, g^{*}\right)$, which, exactly as before, must be Ricci-flat, homogeneous, and hence flat. In particular, it must be isometric to $\mathbb{R}^{n-k} \times T^{k}$, where $T^{k}$ is a flat-torus (see Theorem 2.7.1 in [Wo2]).

We next rule out the case $k=0$ by making use of a very special property of the harmonic norm (cf. (4) on p. 304 of [Pe2]), namely, that

$$
\|A \subset M, g\|_{1, \alpha, r}^{h}=\sup _{x \in A}\|\{x\} \subset M, g\|_{1, \alpha, r}^{h} .
$$

Applying this together with the compactness and homogeneity of $M$, we obtain

$$
\left\|\left\{x_{0}\right\} \subset M, \hat{g}_{i}\right\|_{1, \alpha, 1}^{h}=\left\|M, \hat{g}_{i}\right\|_{1, \alpha, 1}^{h}=1 .
$$

On the other hand, by (3) on p. 304 of [Pe2], we also have

$$
\left\|\left\{x^{*}\right\} \subset M^{*}, g^{*}\right\|_{1, \alpha, 1}^{h}=\lim _{i \rightarrow+\infty}\left\|\left\{x_{0}\right\} \subset M, \hat{g}_{i}\right\|_{1, \alpha, 1}^{h}=1 .
$$

Recall that the linear functions give global coordinates on Euclidean space $\mathbb{R}^{n}$, and so the harmonic norm $\left\|\{0\} \subset \mathbb{R}^{n}, g_{\text {can }}\right\|_{1, \alpha, 1}^{h}=0$. Therefore $\left(M^{*}, g^{*}\right)$ must have a compact torus factor, and in particular non-trivial geodesic loops. With the help of simple geometric arguments we will conclude below that for all but finitely many $i$ the isotropy representation of $H_{i}$ on the tangent space $\mathfrak{m}^{i}$ of $G_{i} / H_{i}$ at $x_{0}$ has non-trivial summands acted on trivially by $H_{i}^{0}$. This in turn implies Theorem 2.1.

Let us assume now that there exist infinitely many $i$ such that the isotropy representation of $H_{i}$ on $\mathfrak{m}^{i}$ does not have non-trivial summands acted on trivially by $H_{i}^{0}$. Since $H_{i}^{0}$ is normal in $H_{i}$, it is easy to see that then $H_{i}^{0}$ does not fix any non-trivial subspace of $\mathfrak{m}^{i}$. As we will see below, this implies that there exists a constant $\alpha(n)>0$ only depending on $n=\operatorname{dim} M$ such that for any unit vector $v \in \mathfrak{m}^{i}$ there exists $h_{i}^{v} \in H_{i}^{0}$ with

$$
\angle\left(v,\left(h_{i}^{v}\right)_{*} \cdot v\right) \geq \alpha(n)>0 .
$$

Let us pick now any geodesic loop $\gamma_{v^{*}}$ of unit speed on $\left(M^{*}, g^{*}\right)$ emanating from $x^{*}$. Since $\left(M^{*}, g^{*}\right)$ is flat, there exists only finitely many geodesic loops of fixed length passing through $x^{*}$. Let $c_{v^{*}}>0$ denote the minimal angle between $v^{*}$ and $w^{*}$ for any other geodesic loop $\gamma_{w^{*}}$ of equal length. Since $\left(M, x_{0}, \tilde{g}_{i}\right)$ converges to $\left(M^{*}, x^{*}, g^{*}\right)$ in $C^{1}$ topology, there exists a sequence of unit speed geodesics $\gamma_{v_{i}}$ on $\left(M, \tilde{g}_{i}\right)$ emanating from $x_{0}$ which converges to $\gamma_{v^{*}}$. Since the isotropy groups $H_{i}$ act on $\mathfrak{m}^{i}$ by isometries fixing $x_{0}$, geodesics emanating from $x_{0}$ are mapped onto such geodesics by $H_{i}$. Therefore, (2.2) implies that there exists $h_{i}^{v_{i}} \in H_{i}^{0}$ such that

$$
\angle\left(v_{i},\left(h_{i}^{v_{i}}\right)_{*} \cdot v_{i}\right)=\min \left\{\frac{c_{v^{*}}}{2}, \alpha(n)\right\} .
$$

After passing to a subsequence we may assume that $\gamma_{\left(h_{i}^{v_{i}}\right)_{*} \cdot v_{i}}$ converges to a geodesic loop $\gamma_{w^{*}}$ emanating from $x^{*}$. Since $\gamma_{v^{*}}$ and $\gamma_{w^{*}}$ are of equal length we obtain a contradiction.

We are left proving the existence of the constant $\alpha(n)$. Let $v_{i} \in \mathfrak{m}^{i}$ be a vector of unit length and let $\mathfrak{m}^{i}=\mathfrak{m}_{1}^{i} \oplus \mathfrak{m}_{2}^{i} \oplus \cdots \oplus \mathfrak{m}_{t_{i}}^{i}$ be a $\tilde{g}_{i}$-orthogonal splitting of $\mathfrak{m}^{i}$ into $\operatorname{Ad}\left(H_{i}^{0}\right)$-irreducible summands. Since $\tilde{g}_{i}\left(v_{i}, v_{i}\right)=1$ and $\operatorname{dim} \mathfrak{m}^{i}=n$ there exist $j \in\left\{1,2, \ldots, t_{i}\right\}$ such that the $\tilde{g}_{i^{-}}$ orthogonal projection of $v_{i}$ onto $\mathfrak{m}_{j}^{i}$ has length bigger or equal than $\frac{1}{n}$. Since $\operatorname{dim} H_{i} \leq \frac{1}{2} n(n+1)$, we can apply Lemma 2.3 and we obtain the claim.

Lemma 2.3. Let $H$ be a compact connected Lie group with $\operatorname{dim} H \leq \frac{1}{2} n(n+1), n \geq 2$. If $H$ acts irreducibly on a real vector space $V$ with $\operatorname{dim} V=n$, then there exists a constant $\alpha(n)>0$ only depending on $n$, such that for all $v \in V \backslash\{0\}$ there exists $h_{*} \in H$ with $\angle\left(v, h_{*} . v\right) \geq \alpha(n)$, where the angle $\angle$ is induced from an $H$-invariant scalar product on $V$. 
Proof. For a fixed action of $H$ there exists $h_{*}$ with $\angle\left(v, h_{*} . v\right)>0$ for all $v \in V \backslash\{0\}$. So it is enough to show that up to orbit equivalence there exist at most finitely many such actions.

We may assume that $H$ acts almost effectively. As is well known there exists a finite covering space of $H$ of the form $T^{k} \times L_{1} \times \cdots \times L_{l}$ where $k \geq 0$ and $L_{1}, \ldots, L_{l}$ are simple, simply connected compact Lie groups. Since the covering map is a Lie group homomorphism, we can lift the action of $H$ to an irreducible action of $T^{k} \times L_{1} \times \cdots \times L_{l}$ on $V$. Next, we extend the action of $H$ to an action on $V \otimes \mathbb{C}$, and the up to a constant uniquely determined $H$-invariant scalar product to a hermitian scalar product on $V \otimes \mathbb{C}$. There are two cases: Either $V \otimes \mathbb{C}$ is irreducible or we have $V \otimes \mathbb{C}=W \oplus W^{*}, W$ acted on irreducibly by $H$. In the first case we have $V \otimes \mathbb{C}=\mathbb{C} \otimes W_{1} \otimes \cdots \otimes W_{l}$, where $W_{i}$ are acted on irreducibly by $L_{i}$ and $\mathbb{C}$ is acted on irreducibly by $T^{r}$. Since there exist only finitely many possibilities for $L_{1}, \ldots, L_{l}$, by the Weyl character formula (cf. [BtD], VI.1.7) there exist only finitely many such irreducible representations $W_{i}, 1 \leq i \leq l$. Furthermore all irreducible representations of $T^{k}$ on $\mathbb{C}$ are orbit-equivalent. Therefore, we have up to orbit equivalence at most finitely many such representations. In the second case $V$ itself can be endowed with a complex structure such that $H$ acts by unitary transformations.

\section{The Graph Theorem}

Let $G / H$ be a connected homogeneous space where $G$ and $H$ are compact. We will obtain a rough picture of the regions in $\mathcal{M}_{1}^{G}$ where the scalar curvature function is negative and the regions where it tends to $+\infty$. This is described in terms of a graph constructed out of the $A d(H)$-invariant subalgebras lying in-between the Lie algebras $\mathfrak{h}$ and $\mathfrak{g}$ of $H$ and $G$ respectively. Using this picture and Proposition 3.7 we will then derive this section's main Theorem 3.3.

Let $\mathfrak{k}$ be a proper subalgebra of $\mathfrak{g}$ of dimension $d$ containing $\mathfrak{h}$ properly. We shall refer to such a $\mathfrak{k}$ as a proper intermediate subalgebra. In the Grassmannian $G_{d}(\mathfrak{g})$ of $d$-dimensional subspaces in $\mathfrak{g}$ we consider the set $\mathcal{A}_{d}$ of all $d$-dimensional $A d(H)$-invariant intermediate Lie subalgebras

of $\mathfrak{g}$. This is a compact subset of $G_{d}(\mathfrak{g})$. We shall denote by $[\mathfrak{k}]$ the connected component of $\mathfrak{k}$ in $\mathcal{A}_{d}$. (The reason for taking the component instead of just $\mathfrak{k}$ is to take care of continuous families of subalgebras.) When $H=\{1\}$, the sets $\mathcal{A}_{d}$ were studied by R. W. Richardson [Ri] in the context of deformations of Lie subalgebras.

Definition 3.1. The graph of $G / H$ consists of vertices $[\mathfrak{k}]$, where $\mathfrak{k}$ ranges over all proper intermediate $A d(H)$-invariant subalgebras, and edges joining $\left[\mathfrak{k}_{1}\right]$ and $\left[\mathfrak{k}_{2}\right]$ whenever some subalgebra in $\left[\mathfrak{k}_{1}\right]$ contains or is contained in some subalgebra in $\left[\mathfrak{k}_{2}\right]$.

The situation when the graph of $G / H$ is empty is also of interest. This is precisely the case when $G / H$ is primitive, i.e., the only $G$-invariant foliations are the foliation by points and the foliation by the whole space (cf. [Go], [WZ5]). Here we note that the proof of Theorem 2.2 in [WZ2] carries over directly to show:

Theorem 3.2. For a primitive homogeneous space $G / H$ with $G$ and $H$ compact, the scalar curvature function is proper and bounded from above, and hence a maximum point is a $G$ invariant Einstein metric.

In the special case when $H$ (and hence also $G$ ) is connected, primitivity is equivalent to $\mathfrak{h}$ being a maximal subalgebra of $\mathfrak{g}$. But, as was observed in [Go] and [WZ5], if $H$ is not connected, many further examples arise. For example, when $G$ and $H$ have the same rank, the primitive homogeneous spaces $G / H$ coincide with the class of isotropy irreducible spaces with equal rank, which include examples where $\mathfrak{h}$ is not maximal in $\mathfrak{g}$ (see [WZ4]). 
We will defer the study of the properties of the graph of $G / H$ to the next section. However, one of the results that we will use in this section is Proposition 4.1 which states that the graph has only finitely many vertices, and hence finitely many components. We will say that a component is non-toral if it contains a vertex $[\mathfrak{k}]$ with a subalgebra $\mathfrak{k}$ such that if we write $\mathfrak{k}=\mathfrak{h} \perp \mathfrak{m}_{\mathfrak{k}}$ then $\left[\mathfrak{m}_{\mathfrak{k}}, \mathfrak{m}_{\mathfrak{k}}\right] \neq 0$. Otherwise we say it is a toral component.

TheOREM 3.3. Let $G / H$ be a compact connected homogeneous space. If the graph of $G / H$ contains at least two non-toral components, then $G / H$ admits a $G$-invariant Einstein metric with positive scalar curvature.

Recall that in section 1 we chose a normal homogeneous metric $Q$ on $G / H$ which serves as a base point of $\mathcal{M}_{1}^{G}$. Note that if $G / H$ is not a torus then $Q$ always has positive scalar curvature. It is shown in [He], p. 226 that each unit speed geodesic emanating from $Q$ has the form

$$
\gamma_{v}(t)=e^{t v_{1}} Q\left|\mathfrak{m}_{1} \perp e^{t v_{2}} Q\right| \mathfrak{m}_{2} \perp \cdots \perp e^{t v_{\ell}} Q \mid \mathfrak{m}_{\ell}
$$

where $\oplus_{i=1}^{\ell} \mathfrak{m}_{i}$ is a decomposition of $\mathfrak{m}$ into $A d(H)$-irreducible and $Q$-orthogonal subrepresentations, and

$$
\left\langle\gamma_{v}^{\prime}(0), \gamma_{v}^{\prime}(0)\right\rangle=\langle v, v\rangle=\sum_{i=1}^{\ell}\left(\operatorname{dim} \mathfrak{m}_{i}\right) v_{i}^{2}=1, \quad \sum_{i=1}^{\ell}\left(\operatorname{dim} \mathfrak{m}_{i}\right) v_{i}=0 .
$$

We will call such a decomposition a good decomposition of $\gamma_{v}$.

Remark 3.5. For a fixed geodesic $\gamma_{v}$ a good decomposition might not be unique, although the set of all good decompositions is a compact set. However, the set of dimensions $\left\{\operatorname{dim} \mathfrak{m}_{i}\right\}$ depends only on $G / H$ and the $v_{1}, v_{2}, \cdots, v_{\ell}$ are uniquely determined up to order. We will therefore regard any splitting of $\mathfrak{m}$ into $A d(H)$-irreducible summands as ordered, i.e., $\mathfrak{m}=\mathfrak{m}_{1} \oplus \mathfrak{m}_{2}$ and $\mathfrak{m}=\mathfrak{m}_{2} \oplus \mathfrak{m}_{1}$ would be considered as two different decompositions.

Next, we have to recall the structure constants $[i j k]$ introduced in [WZ2], which are totally symmetric in $i, j, k$. Choose $Q$-orthonormal bases $\left\{X_{\mu}\right\},\left\{Y_{\nu}\right\},\left\{Z_{\rho}\right\}$ of $\mathfrak{m}_{i}, \mathfrak{m}_{j}, \mathfrak{m}_{k}$ respectively. Then

$$
[i j k]:=\sum_{\mu, \nu, \rho} Q\left(\left[X_{\mu}, Y_{\nu}\right], Z_{\rho}\right)^{2} .
$$

Note that $[i j k]$ are non-negative continuous functions on the space of all $Q$-orthogonal ordered decompositions of $\mathfrak{m}$ into $A d(H)$-irreducible summands. We have [WZ2]

$$
S\left(\gamma_{v}(t)\right)=\frac{1}{2} \sum_{i=1}^{\ell} d_{i} b_{i} \cdot e^{t\left(-v_{i}\right)}-\frac{1}{4} \sum_{i, j, k=1}^{\ell}[i j k] \cdot e^{t\left(v_{i}-v_{j}-v_{k}\right)}
$$

where the non-negative constants $b_{i}$ are the ratios of the negative of the Killing form on $\mathfrak{g}$ to the bi-invariant metric $Q$ on the respective summands $\mathfrak{m}_{i}$.

In order to prove the Graph Theorem we will show that the following proposition is applicable, which is an immediate consequence of Theorem 1.1 and standard arguments in critical point theory (cf. $[\mathrm{Pa}]$ ).

Proposition 3.7. Let $G$ and $H$ be compact Lie groups such that $G / H$ is a connected, compact homogeneous space. Suppose that there exists a constant $A>0$ such that $\{S \geq A\}$ is disconnected in the space $\mathcal{N}_{1}^{G}$, and furthermore that two of its connected components can be connected by a continuous curve $c:[0,1] \longrightarrow \mathcal{M}_{1}^{G}$ lying in $\{0<S \leq A\}$. Then $G / H$ carries a $G$-homogeneous Einstein metric for the same transitive action with scalar curvature lying in $\left[\min _{t}\{S(c(t))\}, A\right]$. 
Proof of Theorem 3.3. Let us choose $\alpha_{0}=0$ and positive real numbers $\alpha_{1}, \ldots, \alpha_{\ell-1}$ and $\delta$ such that $\alpha_{\ell-1}=1, \alpha_{p}-\alpha_{p-1}>\delta$, and

$$
2\left(\alpha_{p}-\delta\right)-\alpha_{p-1}>1
$$

Given a unit speed geodesic $\gamma_{v}$ in $\mathcal{M}_{1}^{G}$ emanating from $Q$, let

$$
v_{\min }:=\min \left\{v_{1}, v_{2}, \ldots, v_{\ell}\right\} \text {. }
$$

Because the equations satisfied by $v_{i}$ involve only the dimensions $d_{i}=\operatorname{dim} \mathfrak{m}_{i}$, for all $\gamma_{v}$ we have $v_{\min } \leq-c<0$ for some positive number $c$ depending only on $G / H$ (cf. (3.4)). Next define the intervals

$$
I_{p}:=\left[\left(\alpha_{p}-\delta\right) v_{\min }, \alpha_{p-1} v_{\min }\right] .
$$

We say that $I_{p}$ is empty (with respect to $\gamma_{v}$ ) if none of the $v_{i}$ 's belong to $I_{p}$. At least one $I_{p}$ must be empty. Notice that an empty interval signifies a jump in the growth rate of the corresponding component (with respect to the good decomposition) of the metrics lying on the geodesic $\gamma_{v}$.

Next, let

$$
J_{p}:=\left\{j \in\{1,2, \cdots, \ell\} \mid v_{j}<\left(\alpha_{p}-\delta\right) v_{\min }\right\} \text { and } \mathfrak{v}_{p}:=\mathfrak{h} \oplus \bigoplus_{j \in J_{p}} \mathfrak{m}_{j} .
$$

In general, $\mathfrak{v}_{p}$ does not have to be a subalgebra of $\mathfrak{g}$, but the cases where $\mathfrak{v}_{p}$ is actually a subalgebra will be of particular interest. Notice $v_{1}, v_{2}, \ldots, v_{\ell}$ and hence $v_{\min }, I_{p}$ and $\mathfrak{v}_{p}$ depend only on $\gamma_{v}$ and not on any decomposition chosen, whereas $J_{p}$ depends on the decomposition since our decompositions are ordered.

Define now $f: \mathcal{M}_{1}^{G} \backslash\{Q\} \rightarrow \mathbb{R}$ as follows:

$$
f\left(\gamma_{v}(t)\right):=\min \left(\sum_{\left\{p \in\{1, \cdots, \ell-1\} \mid I_{p} \text { empty }\right\}} \sum_{i, j \in J_{p}, k \notin J_{p}}[i j k]\right)
$$

where $t>0$ and the minimum is taken over the compact set of good decompositions for $\gamma_{v}$. Then $f$ is lower semi-continuous. To see this, suppose that $\gamma_{v_{\beta}}$ converges to $\gamma_{v}$. We choose a good decomposition for each $\gamma_{v_{\beta}}$ such that the minimum in the definition of $f\left(\gamma_{v_{\beta}}\right)$ is assumed. By passing to a subsequence we can assume that these decompositions converge to a good decomposition for $\gamma_{v}$. Since $v_{i}^{\beta} \rightarrow v_{i}, I_{p}^{\beta} \rightarrow I_{p}, J_{p}^{\beta} \rightarrow J_{p},[i j k]^{\beta} \rightarrow[i j k]$, and since the $I_{p}$ 's are closed it follows that $\underline{\lim } f\left(\gamma_{v_{\beta}}\right) \geq f\left(\gamma_{v}\right)$.

For $t>0$ let $\Sigma$ and $B(t)$ denote respectively the sphere of radius 1 and the open ball of radius $t$ around $Q$, respectively. Also, let $\Gamma_{i}$ denote a component of the graph of $G / H$. Corresponding to $\Gamma_{i}$, we define $W_{i}^{\Sigma}$ to be the set of all metrics $g \in \Sigma$ such that whenever $I_{p}$ is empty for some $p$, then $\mathfrak{v}_{p}$ is a subalgebra of $\mathfrak{g}$ whose associated vertex $\left[\mathfrak{v}_{p}\right] \in \Gamma_{i}$. Notice that for $g \in \Sigma$ we have $f(g)=0$ iff $g \in \bigcup_{i} W_{i}^{\Sigma}$.

LEMma 3.8. The set $W_{i}^{\Sigma}$ is a non-empty, compact subset of $\Sigma$. Furthermore, $W_{i}^{\Sigma} \cap W_{j}^{\Sigma}=\emptyset$ iff $i \neq j$. If the graph of $G / H$ has at least two components, then $\Sigma \neq \bigcup_{i} W_{i}^{\Sigma}$.

Proof. Let $[\mathfrak{k}]$ be a vertex in $\Gamma_{i}$. Then $\mathfrak{k}=\mathfrak{h} \oplus \mathfrak{m}_{\mathfrak{k}}$ and $\mathfrak{g}=\mathfrak{k} \oplus \mathfrak{m}_{\mathfrak{k}}^{\perp}$ with $Q\left(\mathfrak{m}_{\mathfrak{k}}, \mathfrak{m}_{\mathfrak{k}}^{\perp}\right)=0$. For appropriate $0<x_{1}<x_{2}$ we have $g=x_{1} Q\left|\mathfrak{m}_{\mathfrak{k}} \perp x_{2} Q\right| \mathfrak{m}_{\mathfrak{k}}^{\perp} \in W_{i}^{\Sigma}$. Thus $W_{i}^{\Sigma} \neq \emptyset$. Now let $g \in W_{i}^{\Sigma} \cap W_{j}^{\Sigma}$. Then for every empty $I_{p},\left[\mathfrak{v}_{p}\right]$ is a vertex in both $\Gamma_{i}$ and $\Gamma_{j}$ and hence $i=j$. Next, let $g_{\beta} \in W_{i}^{\Sigma}$ converge to $g \in \Sigma$. Let $I_{p}$ be an empty interval for $g$. Then for sufficiently large $\beta$, $I_{p}^{\beta}$ will also be empty and $\mathfrak{v}_{p}^{\beta}$ converges to $\mathfrak{v}_{p}$. Hence $\mathfrak{v}_{p}$ is a subalgebra of $\mathfrak{g}$. By Proposition 4.1 we can assume $\left[\mathfrak{v}_{p}^{\beta}\right]=\left[\mathfrak{v}_{p}\right]$ for sufficiently large $\beta$. Thus $\left[\mathfrak{v}_{p}\right]$ is a vertex of $\Gamma_{i}$ and we obtain the 
compactness of $W_{i}^{\Sigma}$. Finally, suppose that the graph of $G / H$ has at least two components. By Proposition 4.1 there are only finitely many $W_{i}^{\Sigma}$. Since they are all closed and pairwise disjoint and $\Sigma$ is connected, we must have $\Sigma \neq \bigcup_{i} W_{i}^{\Sigma}$.

We can now state the key uniform scalar curvature estimate which gives a rough picture of the positive and negative regions of the scalar curvature function on $\mathcal{M}_{1}^{G}$. To do this we choose disjoint open neighborhoods $U_{i}^{\Sigma}$ of $W_{i}^{\Sigma}$ in $\Sigma$. Let $C\left(U_{i}^{\Sigma}\right):=\left\{\gamma_{v}(t) \mid t \geq 1, \gamma_{v}(1) \in U_{i}^{\Sigma}\right\}$ and define $C\left(W_{i}^{\Sigma}\right)$ using $W_{i}^{\Sigma}$ in place of $U_{i}^{\Sigma}$. Finally, let $C\left(U^{\Sigma}\right)=\bigcup_{i} C\left(U_{i}^{\Sigma}\right)$. The truncated cones $C\left(U_{i}^{\Sigma}\right)$ are disjoint open neighborhoods of $C\left(W_{i}^{\Sigma}\right)$ in $\mathcal{M}_{1}^{G} \backslash B(1)$, and since $\Gamma$ has at least two non-toral components $\mathcal{M}_{1}^{G} \backslash\left\{C\left(U^{\Sigma}\right) \cup B(1)\right\}$ is non-empty.

Proposition 3.9. There exists $t_{0} \geq 1$ such that if $g \in \mathcal{M}_{1}^{G} \backslash\left\{C\left(U^{\Sigma}\right) \cup B\left(t_{0}\right)\right\}$ then $S(g)<0$.

Proof. Let $g \in \mathcal{M}_{1}^{G} \backslash B(1)$. Since $f(g)=0$ iff $g \in \bigcup_{i} C\left(W_{i}^{\Sigma}\right), f$ must be positive on $\mathcal{M}_{1}^{G} \backslash\left\{C\left(U^{\Sigma}\right) \cup B(1)\right\}$. In fact, on this set $f$ is uniformly bounded below by a positive constant $y_{0}$ because $f$ is lower semi-continuous and constant along each $\gamma_{v}$. This implies the existence of $y_{1}>0$ such that for any $g \in \mathcal{M}_{1}^{G} \backslash\left\{C\left(U^{\Sigma}\right) \cup B(1)\right\}$ there exists an empty $I_{p}$, and indices $i, j \in J_{p}, k \notin J_{p}$ with $[i j k]>y_{1}$. We may now proceed as on p. 184 of [WZ2] in order to obtain the existence of $t_{0}$.

Finally, let us fix a non-toral component $\Gamma_{i}$ of the graph of $G / H$ and let $[\mathfrak{k}] \in \Gamma_{i}$ such that $\left[\mathfrak{m}_{\mathfrak{k}}, \mathfrak{m}_{\mathfrak{k}}\right] \neq 0\left(\mathfrak{k}=\mathfrak{h} \oplus \mathfrak{m}_{\mathfrak{k}}\right)$. As in the proof of Lemma 3.8 we define the unit speed geodesic $\gamma_{v}(t)=e^{t v_{1}} Q\left|\mathfrak{m}_{\mathfrak{k}} \perp e^{t v_{2}} Q\right| \mathfrak{m}_{\mathfrak{k}}^{\perp}$ for appropriate choices of $v_{1}<0<v_{2}$. Next, let $\oplus_{i=1}^{\ell} \mathfrak{m}_{i}$ be a good decomposition of $\gamma_{v}$ compatible with the splitting $\mathfrak{m}=\mathfrak{m}_{\mathfrak{k}} \oplus \mathfrak{m}_{\mathfrak{k}}^{\perp}$. Suppose that $\mathfrak{m}_{\mathfrak{k}}=\oplus_{i=1}^{\ell_{1}} \mathfrak{m}_{i}$. Let $a_{0}^{i}=d_{i} b_{i}-\frac{1}{2} \sum_{j, k=1}^{\ell_{1}}[i j k]-\sum_{j, k=\ell_{1}+1}^{\ell}[i j k], a_{1}=\sum_{i=\ell_{1}+1}^{\ell}\left(d_{i} b_{i}-\frac{1}{2} \sum_{j, k=\ell_{1}+1}^{\ell}[i j k]\right)$ and $a_{2}=\frac{1}{2} \sum_{i=1}^{\ell_{1}} \sum_{j, k=\ell_{1}+1}^{\ell}[i j k]$. Then from (3.6) we deduce that for $t \in[0,+\infty)$

$$
2 S\left(\gamma_{v}(t)\right)=e^{-t v_{1}}\left(\sum_{i=1}^{\ell_{1}} a_{0}^{i}+a_{1} x(t)-a_{2} x^{2}(t)\right)
$$

where $x(t):=e^{t\left(v_{1}-v_{2}\right)}$ takes values in the interval $(0,1]$. Observe that $2 S\left(\gamma_{v}(0)\right)=2 S(Q)=$ $\sum_{i=1}^{\ell_{1}} a_{0}^{i}+a_{1}-a_{2}>0$, since otherwise $G / H$ would be a torus and its graph would be connected. With the help of equation (1.5) in [WZ2] we conclude that

$$
a_{0}^{i}=2 d_{i} c_{i}+\frac{1}{2} \sum_{j, k=1}^{\ell_{1}}[i j k] \geq 0,
$$

where $c_{i} \geq 0$ is the eigenvalue of the Casimir operator of the $A d(H)$-representation $\mathfrak{m}_{i}$. Hence $a_{0}:=\sum_{i=1}^{\ell_{1}} a_{0}^{i}>0$, which implies that $\lim _{t \rightarrow+\infty} S\left(\gamma_{v}(t)\right)=+\infty$.

Now the parabola $p(x)=a_{0}+a_{1} x-a_{2} x^{2}$ satisfies $p(0), p(1)>0$, and since $a_{2} \geq 0$ we have $p(x)>0$ for all $x \in(0,1]$. Hence, if there are two non-toral components in the graph, the above construction gives two geodesics $\gamma_{i}(i=1,2)$ emanating from $Q$ such that $\gamma_{i}(t) \in C\left(W_{i}^{\Sigma}\right)$ for $t \geq 1, S\left(\gamma_{i}(t)\right)>0$ for $t \geq 0$, and $S\left(\gamma_{i}(t)\right) \rightarrow+\infty$ as $t \rightarrow+\infty$. The hypotheses of Proposition 3.7 are therefore satisfied, and existence of a $G$-invariant Einstein metric follows.

Finally we mention the following extension of Theorem 3.3 which follows from Corollary 4.8.

Proposition 3.10. Let $G / H$ be a compact homogeneous space with finite fundamental group such that both $G$ and $H$ are connected. If the graph of $G / H$ contains at least two components, then $G / H$ admits a $G$-invariant Einstein metric with positive scalar curvature. 


\section{Properties of the Graph of $G / H$}

In this section we will derive various properties of the graph of an effective connected homogeneous space $G / H$, where $G$ is a compact Lie group and $H$ is a closed subgroup. Some of the properties will be useful for finding homogeneous spaces with disconnected graph (see section 6). We shall also give an application to the study of manifolds admitting infinitely many transitive actions (Proposition 4.6).

Recall that we introduced the compact subset $\mathcal{A}_{d}$ of the Grassmannian $G_{d}(\mathfrak{g})$ consisting of all those $d$-dimensional subspaces which are proper $A d(H)$-invariant intermediate subalgebras of $\mathfrak{g}$. Note that when $H$ is connected, any intermediate subalgebra is automatically $A d(H)$-invariant.

Proposition 4.1. The set $\mathcal{A}_{d}$ has only finitely many connected components, each one of which is compact. The graph of $G / H$ has only finitely many vertices and therefore only finitely many components.

Proof. We will show that $\mathcal{A}_{d}$ is an algebraic subvariety of $G_{d}(\mathfrak{g})=O(N) /(O(d) \times O(N-d))$, where $N=\operatorname{dim} \mathfrak{g}$, and orthogonality is defined using the bi-invariant metric $Q$. It then follows from a classical theorem of $\mathrm{H}$. Whitney [Wh], p. 547, that there are only a finite number of components.

We first assume that $H$ is connected. We fix a $Q$-orthonormal basis $\left\{X_{1}, \cdots, X_{\ell}\right\}$ of $\mathfrak{h}$. For any ordered $Q$-orthonormal basis $\left\{Y_{1}, \cdots, Y_{N}\right\}$ of $\mathfrak{g}$, define the functions $\tilde{F}_{i}$ on $\mathrm{O}(N)$ as follows:

$$
\begin{aligned}
& \tilde{F}_{1}\left(Y_{1}, \cdots, Y_{N}\right):=\sum_{1 \leq \alpha, \beta \leq d, d+1 \leq j \leq N} Q\left(\left[Y_{\alpha}, Y_{\beta}\right], Y_{j}\right)^{2}, \\
& \tilde{F}_{2}\left(Y_{1}, \cdots, Y_{N}\right):=\sum_{1 \leq k \leq \ell, d+1 \leq j \leq N} Q\left(Y_{j}, X_{k}\right)^{2} .
\end{aligned}
$$

Clearly, $\tilde{F}_{i}$ are polynomial functions in the entries of the orthogonal matrix determined by $\left\{Y_{1}, \cdots, Y_{N}\right\}$ depending only on the linear span of $\left\{Y_{1}, \cdots, Y_{d}\right\}$ and of $\left\{Y_{d+1}, \cdots, Y_{N}\right\}$. Therefore they determine functions $F_{1}, F_{2}: G_{d}(\mathfrak{g}) \longrightarrow \mathbb{R}$. Now observe that $\mathcal{A}_{d}=F_{1}^{-1}(0) \cap F_{2}^{-1}(0)$.

If $H$ is not connected, we select representatives $h_{1}, \cdots, h_{e}$ of $A d(H) / A d\left(H_{0}\right)$ and define

$$
\tilde{F}_{i+2}\left(Y_{1}, \cdots, Y_{N}\right):=\sum_{1 \leq \alpha \leq d, d+1 \leq j \leq N} Q\left(\operatorname{Ad}\left(h_{i}\right)\left(Y_{\alpha}\right), Y_{j}\right)^{2}, \quad 1 \leq i \leq e .
$$

Then $\mathcal{A}_{d}=\bigcap_{i=1}^{i=e+2} F_{i}^{-1}(0)$.

Our next goal is to study the structure of a vertex $[\mathfrak{k}]$. These results will be useful for finding homogeneous spaces $G / H$ with disconnected graph, but may be of independent interest in other geometric problems involving families of subgroups or subalgebras. In the remainder of this section we will assume that $G / H$ has finite fundamental group. We will denote the identity component of a compact Lie group $K$ by $K^{0}$ and when we refer to the rank or semisimplicity of $K$ we actually mean the corresponding property of $K^{0}$.

We need to introduce some notation. For any proper intermediate subalgebra $\mathfrak{h} \subset \mathfrak{f} \subset \mathfrak{g}$, let $\mathfrak{h}_{s}$ and $\mathfrak{f}_{s}$ denote the semisimple parts of $\mathfrak{h}$ and $\mathfrak{f}$ respectively, and $\mathfrak{c}\left(\mathfrak{f}_{s}\right)$ denote the centralizer of $\mathfrak{f}_{s}$ in $\mathfrak{g}$. Both $\mathfrak{f}_{s}$ and $\mathfrak{c}\left(\mathfrak{f}_{s}\right)$ are $A d(H)$-invariant, and using the background bi-invariant metric $Q$ we have an $A d(H)$-invariant orthogonal decomposition $\mathfrak{g}=\mathfrak{c}\left(\mathfrak{f}_{s}\right) \oplus \mathfrak{c}\left(\mathfrak{f}_{s}\right)^{\perp}$, where $\mathfrak{f}_{s} \subset \mathfrak{c}\left(\mathfrak{f}_{s}\right)^{\perp}$ and the center $\mathfrak{z}(\mathfrak{f}) \subset \mathfrak{c}\left(\mathfrak{f}_{s}\right)$. While $\mathfrak{h}_{s} \subset \mathfrak{f}_{s}$, the center of $\mathfrak{h}$ in general has a non-trivial projection into $\mathfrak{c}\left(\mathfrak{f}_{s}\right)$, which we will denote by $\mathfrak{a}_{\mathfrak{f}}$ and which is easily seen to be $A d(H)$-invariant. Let $\mathfrak{b}_{\mathfrak{f}}$ be a maximal abelian subalgebra of $\mathfrak{c}\left(\mathfrak{f}_{s}\right)$ containing $\mathfrak{z}(\mathfrak{f})$. We will denote by $F_{s}, C\left(F_{s}\right)^{0}, A_{\mathfrak{f}}$, and $B_{\mathfrak{f}}$ the analytic subgroups in $G$ with Lie algebras $\mathfrak{f}_{s}, \mathfrak{c}\left(\mathfrak{f}_{s}\right), \mathfrak{a}_{\mathfrak{f}}$, and $\mathfrak{b}_{\mathfrak{f}}$ respectively. The first 
two groups and $B_{\mathfrak{f}}$ are obviously compact. To see that the third group is compact, consider the multiplication map $\mu: F_{s} \times B_{\mathfrak{f}} \longrightarrow F_{s} \cdot B_{\mathfrak{f}}$, which is a finite covering map. Since $H$ is a compact subgroup of the compact group $F_{s} \cdot B_{\mathfrak{f}}, \mu^{-1}(H)$ is compact. The same is true of the projection of $\mu^{-1}(H)$ onto $B_{\mathfrak{f}}$. But this is precisely the group $A_{\mathfrak{f}}$.

Starting from an $A d(H)$-invariant intermediate subalgebra $\mathfrak{k}$ as above, one can construct other subalgebras in the same vertex as $\mathfrak{k}$ in two ways. First, the identity component of the normalizer $N(H)$ certainly acts on $\mathfrak{k}$ to give possibly another element of [ $\mathfrak{k}]$. Second, consider $\mathfrak{a}_{\mathfrak{k}} \subset \mathfrak{z}(\mathfrak{k}) \subset \mathfrak{c}\left(\mathfrak{k}_{s}\right)$. If $\mathfrak{z}(\mathfrak{k})$ lies in a continuous family of $A d(H)$-invariant abelian subalgebras in $\mathfrak{c}\left(\mathfrak{k}_{s}\right)$ containing $\mathfrak{a}_{\mathfrak{k}}$, then the direct sums of these abelian subalgebras with $\mathfrak{k}_{s}$ belong to the vertex of $\mathfrak{k}$. Therefore, let $\mathcal{T}_{\mathfrak{k}}$ denote the set of all $A d(H)$-invariant abelian subalgebras of $\mathfrak{c}\left(\mathfrak{k}_{s}\right)$ containing $\mathfrak{a}_{\mathfrak{k}}$ and having the same dimension as $\mathfrak{z}(\mathfrak{k})$, let $\mathcal{T}_{\mathfrak{k}}^{0}$ denote the connected component of $\mathcal{T}_{\mathfrak{k}}$ containing $\mathfrak{z}(\mathfrak{k})$, and let $\mathfrak{k}_{s} \perp \mathcal{T}_{\mathfrak{k}}^{0}$ denote the set of subalgebras of the form $\mathfrak{k}_{s} \perp \mathfrak{t}$ where $\mathfrak{t} \in \mathcal{T}_{\mathfrak{k}}^{0}$. Note that a subalgebra $\mathfrak{t}$ of $\mathfrak{c}\left(\mathfrak{k}_{s}\right)$ contains $\mathfrak{a}_{\mathfrak{k}}$ iff $\mathfrak{k}_{s} \perp \mathfrak{t}$ contains $\mathfrak{h}$.

Proposition 4.2. Let $\mathfrak{k}$ be a proper Ad(H)-invariant intermediate subalgebra. Then

$$
[\mathfrak{k}]=N(H)^{0} \cdot\left(\mathfrak{k}_{s} \perp \mathcal{T}_{\mathfrak{k}}^{0}\right) \text {. }
$$

Furthermore, if $H$ is connected, then

$$
[\mathfrak{k}]=N(H)^{0} \cdot\left(\mathfrak{k}_{s} \perp \mathcal{T}_{\mathfrak{k}}\right) .
$$

The proof of the above proposition uses the following theorem of Montgomery and Zippin ([MZ], p. 216). Let $G^{*}$ be a Lie group and $U$ a given neighborhood of 1 in $G^{*}$. Suppose $K^{*}$ is a compact subgroup of $G^{*}$. Then there exists a neighborhood of $K^{*}$ such that any compact subgroup of $G^{*}$ lying in it is conjugate by an element in $U$ to a subgroup of $K^{*}$. We may assume that the neighborhood of $K^{*}$ is of the form $A d\left(U^{\prime}\right) \cdot K^{*}$ for some neighborhood $U^{\prime} \subset U$ of 1 . Since we will also be dealing with subalgebras, we require the following consequences of this theorem.

Lemma 4.3. Let $\mathfrak{k}$ be a semisimple subalgebra of $\mathfrak{g}$ of dimension $d^{\prime}$ and $U$ be a given neighborhood of 1 in $G$. Then there exists a neighborhood $\mathcal{V}$ of $\mathfrak{k}$ in $G_{d^{\prime}}(\mathfrak{g})$ such that whenever $\mathfrak{l}$ is a subalgebra of $\mathfrak{g}$ lying in $\mathcal{V}$, then there exists an element $u \in U$ so that $A d_{u}(\mathfrak{l})=\mathfrak{k}$.

Proof. Since $\mathfrak{k}$ is semisimple, $K$ is compact, so we can select a neighborhood $U^{\prime}$ of 1 in $G$ according to the theorem of Montgomery-Zippin stated above. If $\mathfrak{l}$ is close to $\mathfrak{k}$, their respective Killing forms $-\kappa_{\mathfrak{k}}$ and $-\kappa_{\mathfrak{l}}$ are both non-degenerate and hence $\mathfrak{l}$ is semisimple and $L$ compact. Since $\operatorname{Ric}\left(\kappa_{\mathfrak{k}}\right)=\frac{1}{4} \kappa_{\mathfrak{k}}$, Myers' theorem implies that the diameter of the metric $\kappa_{\mathfrak{k}}$ is $\leq 2 \pi \sqrt{\operatorname{dim} K-1}$ and hence the exponential map maps $\left\{X \in \mathfrak{k} \mid \kappa_{\mathfrak{k}}(X, X) \leq 2 \pi \sqrt{\operatorname{dim} G-1}\right\}$ onto $K$ and similarly for $L$. So if $\mathfrak{l}$ is sufficiently close to $\mathfrak{k}$, then $L$ lies in $\operatorname{Ad}\left(U^{\prime}\right) \cdot K$ and the lemma follows.

Note that the above lemma does not hold if we merely assume that $\mathfrak{k}$ is a compact Lie algebra.

Lemma 4.4. Let $\mathfrak{k}$ be a subalgebra of $\mathfrak{g}$ of dimension $d$ and let $d^{\prime}=\operatorname{dim}\left(\mathfrak{k}_{s}\right)$. Given any neighborhood $\mathcal{V}$ of $\mathfrak{k}_{s}$ in $G_{d^{\prime}}(\mathfrak{g})$, there exists a neighborhood $\mathcal{W}$ of $\mathfrak{k}$ in $G_{d}(\mathfrak{g})$ such that if $\mathfrak{l}$ is a subalgebra lying in $\mathcal{W}$ then $\operatorname{dim}\left(\mathfrak{l}_{s}\right)=d^{\prime}$ and $\mathfrak{l}_{s}$ lies in $\mathcal{V}$.

Proof. This will follow once we show that if $\mathfrak{l}^{i}$ is a sequence of subalgebras converging to $\mathfrak{k}$, then there exists a subsequence of $\mathfrak{l}^{i}$ such that $\mathfrak{l}_{s}^{i}=\left[\mathfrak{l}^{i}, \mathfrak{l}^{i}\right]$ converges to $\mathfrak{k}_{s}=[\mathfrak{k}, \mathfrak{k}]$. By passing to a subsequence we may assume that $\left[\mathfrak{l}^{i}, \mathfrak{l}^{i}\right]$ all have the same dimension and converge to a 
subalgebra $\mathfrak{l}^{\prime}$ of $\mathfrak{k}$. Clearly $[\mathfrak{k}, \mathfrak{k}] \subset \mathfrak{l}^{\prime}$. To see that $\mathfrak{l}^{\prime} \subset[\mathfrak{k}, \mathfrak{k}]$, let $\left\{e_{j}^{i}\right\}$ be a $Q$-orthonormal basis of $\mathfrak{l}^{i}$ converging to a basis $\left\{e_{j}\right\}$ of $\mathfrak{k}$. Choose a basis $\tilde{\Pi}^{i}$ of $\left[\mathfrak{l}^{i}, \mathfrak{l}^{i}\right]$ among the vectors $\left\{\left[e_{j_{1}}^{i}, e_{j_{2}}^{i}\right], j_{1}<j_{2}\right\}$ and use the Gram-Schmidt process to replace $\tilde{\Pi}^{i}$ by a $Q$-orthonormal basis $\Pi^{i}$ whose elements are therefore finite linear combinations of the elements of $\tilde{\Pi}^{i}$. By taking a further subsequence, we may assume that $\Pi^{i}$ converges to a basis of $\mathfrak{l}^{\prime}$ which are linear combinations of $\left\{\left[e_{j_{1}}, e_{j_{2}}\right]\right\}$. Hence $\mathfrak{l}^{\prime} \subset[\mathfrak{k}, \mathfrak{k}]$ and so $\left[\mathfrak{l}^{i}, \mathfrak{l}^{i}\right]$ converges to $[\mathfrak{k}, \mathfrak{k}]$ as desired.

Proof of Proposition 4.2. Let

$$
\mathcal{O}_{\mathfrak{k}}:=N(H)^{0} \cdot\left(\mathfrak{k}_{s} \perp \mathcal{T}_{\mathfrak{k}}^{0}\right) .
$$

Clearly, by construction $\mathcal{O}_{\mathfrak{k}} \subset[\mathfrak{k}]$. Conversely, we shall show that for every subalgebra $\mathfrak{l} \in \mathcal{O}_{\mathfrak{k}}$ there exists a neighborhood $\mathcal{W}^{*}$ of $\mathfrak{l}$ in $G_{d}(\mathfrak{g})$ such that $\mathcal{A}_{d} \cap \mathcal{W}^{*} \subset \mathcal{O}_{\mathfrak{k}}$. On the other hand, since $\mathcal{T}_{\mathfrak{k}}^{0}$ and $N(H)^{0}$ are compact, $\mathcal{O}_{\mathfrak{k}}$ must be closed and hence must be all of $[\mathfrak{k}]$.

Suppose that $\mathfrak{l} \in \mathcal{O}_{\mathfrak{k}}$. Then there is some $x \in N(H)^{0}$ such that $\mathfrak{l}_{s}=A d_{x^{-1}}\left(\mathfrak{k}_{s}\right)$ and $\mathfrak{z}(\mathfrak{l})=$ $A d_{x^{-1}}(\mathfrak{t})$ where $\mathfrak{a}_{\mathfrak{k}} \subset \mathfrak{t} \subset \mathfrak{c}\left(\mathfrak{k}_{s}\right)$ and $\operatorname{dim} \mathfrak{t}=d-d^{\prime}$. Let us fix a maximal abelian subalgebra $\mathfrak{b}_{\mathfrak{l}} \subset \mathfrak{c}\left(\mathfrak{l}_{s}\right)$ containing $\mathfrak{z}(\mathfrak{l})$. Let $N\left(L_{s}\right)$ denote the normalizer of $L_{s}$ in $G$. Since $\mathfrak{l}_{s}$ is $A d(H)$ invariant, it follows that $H \subset N\left(L_{s}\right)$.

Using the theorem of Montgomery-Zippin and Lemmas 4.3 and 4.4 we make the following choices in the indicated order.

i) Let $U_{0}$ be a neighborhood of 1 in G which intersects $N(H)$ only in $N(H)^{0}$ and which satisfies $U_{0} \cdot U_{0} \subset U_{0}$. For $H \subset N\left(L_{s}\right)$ choose a neighborhood $U_{1} \subset U_{0}$ of 1 in $G$ such that any compact subgroup of $N\left(L_{s}\right)$ lying in $\left(A d\left(U_{1}\right) \cdot H\right) \cap N\left(L_{s}\right)$ is conjugate by an element of $U_{0} \cap N\left(L_{s}\right)$ to a subgroup of $H$.

ii) Let $U_{2}$ be a neighborhood of 1 in $G$ satisfying $U_{2} \cdot U_{2} \subset U_{1}$. For $B_{\mathfrak{l}} \subset C\left(L_{s}\right)^{0}$ select a neighborhood $U_{3} \subset U_{2}$ of 1 in $G$ such that any compact subgroup of $C\left(L_{s}\right)^{0}$ lying in $\mathcal{U}:=$ $\operatorname{Ad}\left(U_{3} \cap C\left(L_{s}\right)^{0}\right) \cdot B_{\mathfrak{l}}$ is conjugate to a subgroup of $B_{\mathfrak{l}}$ by an element in $U_{2} \cap C\left(L_{s}\right)^{0}$.

iii) Pick a relatively compact neighborhood $U_{4} \subset U_{3}$ of 1 in $G$ and a neighborhood $\mathcal{V}$ of $\mathfrak{l}_{s}$ in $G_{d^{\prime}}(\mathfrak{g})$ such that for any subalgebra $\tilde{\mathfrak{l}} \in \mathcal{V}$ one can find $w \in U_{4}$ such that $A d_{w}(\tilde{\mathfrak{l}})=\mathfrak{l}_{s}$.

iv) Choose a neighborhood $\mathcal{W} \subset A d\left(U_{3}\right) \cdot \mathfrak{l}$ of $\mathfrak{l}$ in $G_{d}(\mathfrak{g})$ such that for any subalgebra $\tilde{\mathfrak{l}} \in \mathcal{W}$, we have $\tilde{\mathfrak{l}}_{s} \in \mathcal{V}$ and $\operatorname{dim}\left(\tilde{\mathfrak{l}}_{s}\right)=d^{\prime}$.

v) Finally, we select a neighborhood $\mathcal{W}^{*}$ of $\mathfrak{l}$ in $G_{d}(\mathfrak{g})$ satisfying $A d\left(U_{4}\right) \cdot \mathcal{W}^{*} \subset \mathcal{W}$ and such that for any subalgebra $\tilde{\mathfrak{l}} \in \mathcal{W}^{*}$ the projection of $A d\left(U_{4}\right) \cdot \mathfrak{z}(\tilde{\mathfrak{l}})$ onto $\mathfrak{c}\left(\mathfrak{l}_{s}\right)$ is contained in $\operatorname{Ad}\left(U_{3} \cap C\left(L_{s}\right)^{0}\right) \cdot \mathfrak{b}_{\mathfrak{l}}$

Now suppose that $\mathfrak{r} \in \mathcal{A}_{d} \cap \mathcal{W}^{*}$. By (v) and (iv) we have $\operatorname{dim}\left(\mathfrak{r}_{s}\right)=d^{\prime}$ and $\mathfrak{r}_{s} \in \mathcal{V}$, and by (iii), for some $w \in U_{4}$ we have $A d_{w}\left(\mathfrak{r}_{s}\right)=\mathfrak{l}_{s}$, which implies $A d_{w}\left(\mathfrak{c}\left(\mathfrak{r}_{s}\right)\right)=\mathfrak{c}\left(\mathfrak{l}_{s}\right)$. By (v), $A d_{w}(\mathfrak{z}(\mathfrak{r})) \subset A d\left(U_{3} \cap C\left(L_{s}\right)^{0}\right) \cdot \mathfrak{b}_{\mathfrak{l}}$, and so we can choose a maximal abelian subalgebra $\mathfrak{b}_{\mathfrak{r}} \supset \mathfrak{z}(\mathfrak{r})$ in $\mathfrak{c}\left(\mathfrak{r}_{s}\right)$ such that $A d_{w}\left(B_{\mathfrak{r}}\right)$ lies in $A d\left(U_{3} \cap C\left(L_{s}\right)^{0}\right) \cdot B_{\mathfrak{l}}$. By (ii) one can find $v \in U_{2} \cap C\left(L_{s}\right)^{0}$ such that $A d_{v w}(B \mathfrak{r})=B_{\mathfrak{l}}, A d_{v w}\left(\mathfrak{r}_{s}\right)=\mathfrak{l}_{s}$, and hence $A d_{v w}\left(\mathfrak{c}\left(\mathfrak{r}_{s}\right)\right)=\mathfrak{c}\left(\mathfrak{l}_{s}\right)$ as well.

Consider next $A d_{v w}(H)$. Since $H \subset N\left(R_{s}\right)$, we have $A d_{v w}\left(N\left(R_{s}\right)\right) \subset A d_{v}\left(N\left(L_{s}\right)\right) \subset N\left(L_{s}\right)$, and so $A d_{v w}(H) \subset N\left(L_{s}\right)$. Furthermore, $v w \in U_{1}$ and (i) implies that we can find $u \in U_{0} \cap N\left(L_{s}\right)$ such that $A d_{u v w}(H) \subset H$. But $u v w \in U_{0}$ and so $u v w \in N(H)^{0}$. It follows that $A d_{\text {uvw }}\left(\mathfrak{r}_{s}\right)=\mathfrak{l}_{s}$ and hence $A d_{\text {uvw }}\left(\mathfrak{c}\left(\mathfrak{r}_{s}\right)\right)=\mathfrak{c}\left(\mathfrak{l}_{s}\right)$. From this we can first conclude that $A d_{u v w}(\mathfrak{z}(\mathfrak{r}))$ is an $A d(H)$ invariant abelian subalgebra of $\mathfrak{c}\left(\mathfrak{l}_{s}\right)$ having the same dimension as $\operatorname{dim}(\mathfrak{z}(\mathfrak{k}))$. Secondly, since $A d_{\text {uvw }}(\mathfrak{z}(\mathfrak{h}))=\mathfrak{z}(\mathfrak{h})$, we also have $A d_{\text {uvw }}\left(\mathfrak{a}_{\mathfrak{r}}\right)=\mathfrak{a}_{\mathfrak{l}}$. Therefore, $\mathfrak{a}_{\mathfrak{l}} \subset A d_{\text {uvw }}(\mathfrak{z}(\mathfrak{r}))$, and we obtain $A d_{\text {xuvw }}(\mathfrak{r}) \in \mathfrak{k}_{s} \oplus \mathcal{T}_{\mathfrak{k}}^{0}$. This completes the proof of Proposition 4.2. 
An immediate consequence of Proposition 4.2 is the following

Corollary 4.5. Suppose that $H$ is connected. Then

(a) Each vertex $[\mathfrak{k}]$ always contains a subalgebra whose corresponding subgroup is compact.

(b) If either $\mathfrak{k}$ is semisimple or $\mathfrak{z}(\mathfrak{k})$ is a maximal abelian subalgebra of $\mathfrak{c}\left(\mathfrak{k}_{s}\right)$, then $[\mathfrak{k}]$ is diffeomorphic to $N(H)^{0} /\left(N(H)^{0} \cap N\left(K^{0}\right)\right)$.

(c) If there are no trivial sub-representations in the isotropy representation of $G / H$, then each vertex $[\mathfrak{k}]$ contains only one subalgebra.

Next, we give an application of the above properties of the graph to the structure of manifolds admitting infinitely many inequivalent transitive actions by a compact connected Lie group $G$. A concrete example of such a homogeneous spaces is $S^{2} \times S^{2 n+1}$ (see [WZ3]).

Proposition 4.6. If $M$ admits infinitely many inequivalent effective transitive actions by $G$ with respective isotropy groups $H_{i}$, then there is a subsequence $H_{i_{k}}$, a positive integer $r$, and closed subgroups $K_{i_{k}}$ such that $H_{i_{k}} \subset K_{i_{k}} \subset G$ and $K_{i_{k}} / H_{i_{k}} \approx T^{r}$, i.e., $G / H_{i_{k}}$ is a homogeneous torus bundle over $G / K_{i_{k}}$.

Proof. If $\operatorname{dim} H_{i}=0$, then $H_{i}$ are isomorphic to some finite group $\Gamma$ and there would be an infinite sequence $\phi_{i}: \Gamma \longrightarrow G$ of monomorphisms such that $\phi_{i}(\Gamma)$ are all non-conjugate in $G$. Since $\Gamma$ is finite and $G$ is compact, by passing to a subsequence, we may assume that $\phi_{i}$ converge to a homomorphism $\phi_{\infty}$, which must be injective since there are no small subgroups in $G$. By the theorem of Montgomery-Zippin applied to $\phi_{\infty}(\Gamma) \subset G$ we immediately obtain a contradiction.

Therefore we have $\operatorname{dim} H_{i}>0$. Consider the graph of $G /\{1\}$. Since there are only finitely many vertices, there is a subsequence of Lie algebras $\mathfrak{h}_{i_{k}}$ which all belong to a single vertex of the graph. To simplify notation, we will use the same index set for the subsequence. By Corollary 4.5 (b), if $\mathfrak{h}_{i}$ are semisimple, then $H_{i}^{0}$ are conjugate in $G$. Without loss of generality, we may assume that $H_{i}^{0}$ have the same identity component $H^{*}$. We claim that there can be only finitely many conjugacy classes of the $H_{i}$. Now $H_{i}$ and $H_{j}$ are conjugate in $G$ iff they are conjugate in $N\left(H^{*}\right)$ iff $H_{i} / H^{*}$ and $H_{j} / H^{*}$ are conjugate in $N\left(H^{*}\right) / H^{*}$. So our claim follows again by the argument in the previous paragraph.

Hence $\mathfrak{h}_{i}$ must be non-semisimple, and by Proposition 4.2 we may assume that $\mathfrak{h}_{i}=\mathfrak{h}_{s} \oplus \mathfrak{t}_{i}$, where $\mathfrak{t}_{i}$ are distinct abelian subalgebras of a fixed dimension of $\mathfrak{c}\left(\mathfrak{h}_{s}\right)$. Let $\mathfrak{g}=\mathfrak{h}_{i} \oplus \mathfrak{m}_{0}^{(i)} \oplus\left(\mathfrak{m}_{0}^{(i)}\right)^{\perp}$ be a $Q$-orthogonal $A d\left(H_{i}^{0}\right)$-invariant decomposition in which $\mathfrak{m}_{0}^{(i)}$ is the subspace of $\mathfrak{h}_{i}^{\perp}$ on which $A d\left(H_{i}^{0}\right)$ acts trivially. We have $\mathfrak{c}\left(\mathfrak{h}_{i}\right)=\mathfrak{t}_{i} \oplus \mathfrak{m}_{0}^{(i)} \subset \mathfrak{c}\left(\mathfrak{h}_{s}\right)$. Since $\mathfrak{c}\left(\mathfrak{h}_{s}\right)$ is a compact Lie algebra, by passing to a subsequence, we may assume that the centraliser $\mathfrak{c}\left(\mathfrak{t}_{i}\right)$ of $\mathfrak{t}_{i}$ in $\mathfrak{c}\left(\mathfrak{h}_{s}\right)$ does not depend on $i$. Note that $\mathfrak{c}\left(\mathfrak{t}_{i}\right)$ is a compact Lie algebra. But since $\mathfrak{m}_{0}^{(i)}$ is characterized by $\left[\mathfrak{h}_{i}, \mathfrak{m}_{0}^{(i)}\right]=0$, it follows that $\mathfrak{t}_{i} \oplus \mathfrak{m}_{0}^{(i)}=\mathfrak{c}\left(\mathfrak{t}_{i}\right)=: \mathfrak{c}$. We have $\mathfrak{t}_{i} \subset \mathfrak{c}$ and since $\mathfrak{t}_{i} \neq \mathfrak{t}_{j}$ for $i \neq j$, it follows that $\mathfrak{t}_{i}$ is a proper subalgebra of $\mathfrak{z}(\mathfrak{c})$. We conclude

$$
\mathfrak{n}\left(\mathfrak{h}_{i}\right)=\mathfrak{h}_{s} \oplus \underbrace{\mathfrak{t}_{i} \oplus\left(\mathfrak{t}_{i}^{\perp} \cap \mathfrak{z}(\mathfrak{c})\right)}_{=\mathfrak{z}(\mathfrak{c})} \oplus \mathfrak{c}_{s} .
$$

Next, let $K$ denote the compact connected Lie group with Lie algebra $\mathfrak{h}_{s} \oplus \mathfrak{z}(\mathfrak{c})$. Since $\mathfrak{h}_{s} \oplus \mathfrak{z}(\mathfrak{c})$ is clearly $A d\left(H_{i}\right)$-invariant, we conclude that the compact Lie group $K_{i}=K \cdot H_{i}$ and $K$ have the same Lie algebra. We obtain

$$
K_{i} / H_{i} \approx K /\left(H_{i} \cap K\right) \approx\left(K / H_{i}^{0}\right) /\left(\left(H_{i} \cap K\right) / H_{i}^{0}\right) .
$$


But $\mathfrak{h}_{s} \oplus \mathfrak{z}(\mathfrak{c})=\mathfrak{h}_{i} \oplus\left(\mathfrak{t}_{i}^{\perp} \cap \mathfrak{z}(\mathfrak{c})\right)$, and so $K / H_{i}^{0}$ is a torus $T^{r}$. Furthermore, $\left(H_{i} \cap K\right) / H_{i}^{0}$ is a finite subgroup of $T^{r}$ and so $K_{i} / H_{i} \approx T^{r}$.

Note that Proposition 4.6 combined with Theorem 1.7 immediately yields the special case of Theorem 2.1 in which the sequence $g_{i}$ consists of Einstein metrics.

The next proposition shows that homogeneous spaces whose graph has a toral component are rather special.

Proposition 4.7. Let $G / H$ be an effective compact homogeneous space with finite fundamental group. If the graph of $G / H$ contains a toral component, then one of the following holds: (a) $G$ is semisimple and $H$ is finite.

(b) $G$ is semisimple, rank $N_{G}\left(H^{0}\right)=\operatorname{rank} G$ and after possibly extending $G$ and $N_{G}\left(H^{0}\right)$ by a finite group $G / N_{G}\left(H^{0}\right)$ is an isotropy irreducible homogeneous space.

Proof. If the graph of $G / H$ has a toral component, then for any subalgebra $\mathfrak{k}$ belonging to a vertex in that component, if we write $\mathfrak{k}=\mathfrak{h} \perp \mathfrak{m}_{\mathfrak{k}}$, then we have $\left[\mathfrak{m}_{\mathfrak{k}}, \mathfrak{m}_{\mathfrak{k}}\right]=0$. Let us fix such a $\mathfrak{k}$ and let $\mathfrak{m}_{0}$ denote the subspace of $\mathfrak{m}$ on which $A d\left(H^{0}\right)$ acts trivially. Since $Q\left(\left[\mathfrak{h}, \mathfrak{m}_{\mathfrak{k}}\right], \mathfrak{m}_{\mathfrak{k}}\right)=0$, it follows that $\mathfrak{m}_{\mathfrak{k}} \subset \mathfrak{m}_{0}$.

Consider next the normalizer $\mathfrak{n}(\mathfrak{h})$ of $\mathfrak{h}$, which is precisely $\mathfrak{h} \perp \mathfrak{m}_{0}$. It is also the Lie algebra of $N_{G}\left(H^{0}\right)^{0}=N_{G^{0}}\left(H^{0}\right)^{0}$. Since $N_{G}\left(H^{0}\right)$ is $A d(H)$-invariant, so is $N_{G}\left(H^{0}\right)^{0}$ and hence $\mathfrak{n}(\mathfrak{h})$.

If $\mathfrak{n}(\mathfrak{h})=\mathfrak{g}$, then by effectiveness of $G / H, \mathfrak{h}=0$, and the fundamental group assumption implies that $\mathfrak{g}$ must be semisimple. We are therefore in case (a).

If $\mathfrak{n}(\mathfrak{h}) \neq \mathfrak{g}$, then $[\mathfrak{n}(\mathfrak{h})]$ is a vertex connected to $[\mathfrak{k}]$ and $\left[\mathfrak{m}_{0}, \mathfrak{m}_{0}\right]=0$. In fact, $\mathfrak{n}(\mathfrak{h})$ is a nonsemisimple maximal $A d(H)$-invariant subalgebra of $\mathfrak{g}$ that is connected to every other vertex in the component containing $[\mathfrak{k}]$.

We claim that $\mathfrak{n}(\mathfrak{h})$ has maximal rank. Consider the centralizer $\mathfrak{c}\left(\mathfrak{m}_{0}\right)$ of $\mathfrak{m}_{0}$ in $\mathfrak{g}$, which has maximal rank and contains $\mathfrak{n}(\mathfrak{h})$. Since $\mathfrak{m}_{0}$ is $A d(H)$-invariant, so is $\mathfrak{c}\left(\mathfrak{m}_{0}\right)$. If $\mathfrak{c}\left(\mathfrak{m}_{0}\right)=\mathfrak{g}$, then $\mathfrak{m}_{0}$ is an $A d(H)$-invariant subspace of the center $\mathfrak{z}(\mathfrak{g})$. It follows that the fundamental group of $G / H$ must be infinite, which is a contradiction. Hence $\mathfrak{c}\left(\mathfrak{m}_{0}\right)=\mathfrak{n}(\mathfrak{h})$.

Now consider $G / N_{G}\left(H^{0}\right)$. It is a quotient of $G / H$ and so it is connected. Let $\Gamma$ be the ineffective kernel. Then any subalgebra $\mathfrak{l}$ lying between $\mathfrak{n}(\mathfrak{h})$ and $\mathfrak{g}$ that is $A d\left(N_{G}\left(H^{0}\right)\right)$-invariant must be $A d(H)$-invariant. By the maximality of $\mathfrak{n}(\mathfrak{h})$, either $\mathfrak{l}=\mathfrak{n}(\mathfrak{h})$ or $\mathfrak{l}=\mathfrak{g}$. In other words, $(G / \Gamma) /\left(N_{G}\left(H^{0}\right) / \Gamma\right)$ is primitive. It is proved in [WZ5] that a primitive homogeneous space $G^{*} / H^{*}$ with $\operatorname{rank} G^{*}=\operatorname{rank} H^{*}$ must be isotropy irreducible, i.e., after possibly extending $G^{*}$ and $H^{*}$ by suitable finite groups, the isotropy representation of the resulting homogeneous space is irreducible. It follows from [WZ4] that $G$ is semisimple.

An explicit example for case (b) is the following: $G=S U(p k) \ltimes \Gamma, p \geq 3, k \geq 2$, and $H=(S U(k) \times \cdots \times S U(k)) \ltimes \Gamma$ where $\Gamma$ is the symmetric group on $p$ objects. Then $N\left(H_{0}\right)^{0}=$ $S(U(k) \times \cdots \times U(k))$ gives rise to a toral component in the graph.

Corollary 4.8. Suppose that $G / H$ has finite fundamental group and both $G$ and $H$ are connected. If the graph of $G / H$ contains a toral component, then $G /\left(N_{G}(H)\right)^{0}$ is a hermitian symmetric space. In particular $G / H$ carries a $G$-invariant Einstein metric.

Proof. We are in case (a) of the above Proposition iff $G$ is locally isomorphic to $S U(2)$. In case (b), since $\mathfrak{n}(\mathfrak{h})=\mathfrak{h} \oplus \mathfrak{m}_{0}$ is a maximal subalgebra of $\mathfrak{g}$ of maximal rank, it follows from [Wo2], p. 281, that $G /\left(N_{G}(H)\right)^{0}$ is an irreducible hermitian symmetric space. Then existence of a $G$-invariant Einstein metric on $G / H$ follows from the work of S. Kobayashi [Kob].

We end this section with the following observation: 
Proposition 4.9. Assume that $G / H$ is an almost effective homogeneous space with finite fundamental group. If $G$ is non-semisimple, then the graph of $G / H$ is non-empty and connected.

Proof. Let $\mathfrak{g}=\mathfrak{g}_{s} \oplus \mathfrak{z}(\mathfrak{g})$ with its center $\mathfrak{z}(\mathfrak{g}) \neq 0$. Since $A d(H)$ acts by automorphisms of $\mathfrak{g}$, it leaves both $\mathfrak{g}_{s}$ and $\mathfrak{z}(\mathfrak{g})$ invariant. Likewise, if we denote by $\mathfrak{h}^{\prime}$ the projection of $\mathfrak{h}$ into $\mathfrak{g}_{s}$ with respect to the above splitting of $\mathfrak{g}$, then $\mathfrak{h}^{\prime}$ is $A d(H)$-invariant. Now consider the $A d(H)$ invariant subalgebra $\mathfrak{k}=\mathfrak{h}^{\prime} \oplus \mathfrak{z}(\mathfrak{g})$. Since $G / H$ is almost effective, it contains $\mathfrak{h}$ properly. If $\mathfrak{k}=\mathfrak{g}$, then $\mathfrak{g}_{s}=\mathfrak{h}^{\prime}=\mathfrak{h}_{s}$ because the semisimple part of $\mathfrak{h}$ projects to 0 in $\mathfrak{z}(\mathfrak{g})$. This would contradict almost effectiveness since $G / H$ is not a torus. So $[\mathfrak{k}]$ is a vertex in the graph of $G / H$.

Now let $[\mathfrak{l}]$ be another vertex in the graph. As before let $\mathfrak{l}^{\prime}$ be its projection into $\mathfrak{g}_{s}$, which will be $A d(H)$-invariant. We then have $A d(H)$-invariant subalgebras $\mathfrak{l} \subset \mathfrak{l}^{\prime} \oplus \mathfrak{z}(\mathfrak{g}) \subset \mathfrak{g}$.

If $\mathfrak{l}^{\prime} \oplus \mathfrak{z}(\mathfrak{g})=\mathfrak{g}$, then as above we have $\mathfrak{l}^{\prime}=\mathfrak{g}_{s}=\mathfrak{l}_{s}$. So $\mathfrak{z}(\mathfrak{l}) \subset \mathfrak{z}(\mathfrak{g})$ is $\operatorname{Ad}(H)$-invariant. $\mathfrak{h}$ is contained in but not equal to $\mathfrak{h}^{\prime} \oplus \mathfrak{z}(\mathfrak{l})$. Otherwise, almost effectiveness implies that $\mathfrak{z}(\mathfrak{l})=0$ and $\mathfrak{l}=\mathfrak{g}_{s}$, which contradicts the assumption that $G / H$ has finite fundamental group. It follows that $\left[\mathfrak{h}^{\prime} \oplus \mathfrak{z}(\mathfrak{l})\right]$ is a vertex of the graph which can be connected to both $[\mathfrak{l}]$ and $[\mathfrak{k}]$.

If $\mathfrak{l}^{\prime} \oplus \mathfrak{z}(\mathfrak{g}) \neq \mathfrak{g}$, then $\left[\mathfrak{l}^{\prime} \oplus \mathfrak{z}(\mathfrak{g})\right]$ is a vertex, and since $\mathfrak{h}^{\prime} \subset \mathfrak{l}^{\prime},[\mathfrak{k}]$ is connected to $\left[\mathfrak{l}^{\prime} \oplus \mathfrak{z}(\mathfrak{g})\right]$, which is connected to $[\mathfrak{l}]$.

Note though that if $G$ is not semisimple and if the fundamental group of $G / H$ is finite, then the semisimple part of $G$ also acts transitively on $G / H$ and the corresponding homogeneous space may have a disconnected graph. For example, the Stiefel manifold $S O(n+2) / S O(n)$ has a disconnected graph, but when written as $(S O(n+2) S O(2)) /(S O(n) \cdot \Delta S O(2))$ its graph consists of a single vertex $[\mathfrak{s o}(n) \oplus \mathfrak{s o}(2) \oplus \mathfrak{s o}(2)]$.

\section{The YamaBe InVARIANT}

Recall that the second variation formula of the Hilbert action at an Einstein metric (other than the constant curvature metrics on standard spheres) shows that the action is locally minimizing in the direction of infinitesimal conformal deformations (cf. [Bes], Theorem 4.60) . This motivated the Yamabe problem, whose solution (cf. [Sc1], [LP]) shows that in a fixed conformal class $c$ the infimum of $\int_{M} S_{g} d \mu_{g}$ over the volume 1 metrics is achieved by a metric of constant scalar curvature called a Yamabe metric. One is then naturally led to consider the variational problem associated to the invariant

$$
y(M)=\sup _{c} \inf _{g \in c, \operatorname{vol}(g)=1} \int_{M} S_{g} d \mu_{g}
$$

where $c$ ranges over all conformal classes of metrics on $M$, with the hope that one could construct Einstein metrics via this variational procedure. This invariant, called the Yamabe invariant of $M$, was studied independently in [Ko] and [Sc2]. It is known from these authors that if $y(M) \leq 0$ and if it is achieved by some metric $g$, then $g$ must be an Einstein metric. However, the corresponding statement when $y(M)>0$ is not known.

Conversely, since an Einstein metric $g$ is automatically a Yamabe metric in its conformal class (cf. [Sc2], Proposition 1.4), it is interesting to ask which Einstein metrics actually realize $y(M)$. Petean $[\mathrm{Pe}]$ recently showed that $y(M) \geq 0$ for all simply connected closed manifolds of dimension $>4$, and hence only Einstein metrics with non-negative scalar curvature have a chance of realizing the Yamabe invariant on such manifolds. We will show below that the Einstein metrics of positive scalar curvature produced by the Graph Theorem cannot realize the Yamabe invariant either.

On a compact homogeneous space $M=G / H$ with finite fundamental group, Theorem 1.7 implies that there always exists a homogeneous Einstein metric with largest scalar curvature 
among homogeneous Einstein metrics of unit volume. Furthermore, by Theorem 1.8, when $M$ admits several or even infinitely many transitive actions, there still must exist such a "best" homogeneous Einstein metric, provided that the moduli space of homogeneous Einstein metrics is non-empty. Hence it is natural to ask whether a "best" homogeneous Einstein metric might realize the Yamabe constant. We will again show that this need not be the case.

Our results follow from applying the following

Proposition 5.1. Let $\left(M, g_{\infty}\right)$ be a closed unit volume Einstein manifold of dimension $n \geq 3$ not conformally equivalent to the round sphere. Suppose that there exists a sequence $g_{i}$ of unit volume metrics with constant scalar curvature $S_{g_{i}}>S_{g_{\infty}}$ converging to $g_{\infty}$ in the $C^{3}$ topology. Then $g_{\infty}$ cannot realize the Yamabe invariant of $M$.

Proof. For each $g_{i}$ as above, let $\lambda\left(g_{i}\right)$ denote the Yamabe invariant of its conformal class, i.e., the infimum of the Hilbert action over unit volume metrics in its conformal class. By the solution of the Yamabe problem, there exists a positive $C^{\infty}$-function $\varphi_{i}$ on $M$ with $\left\|\varphi_{i}\right\|_{L^{p}\left(g_{i}\right)}=1$ satisfying the equation

$$
a \cdot \Delta_{g}(\varphi)+S(g) \cdot \varphi=\lambda(g) \cdot \varphi^{p-1},
$$

with $a=\frac{4(n-1)}{n-2}, p=\frac{2 n}{n-2}$ and $g=g_{i}$. (We remind the reader that the norm $\left\|\varphi_{i}\right\|_{L^{p}\left(g_{i}\right)}$ is precisely the volume of the metric $\varphi_{i}^{p-2} g_{i}$.)

If we can show that $\varphi_{i}$ are uniformly bounded in $C^{2, \beta}$ for some $\beta \in(0,1)$ (with respect to $\left.g_{\infty}\right)$, then by the Arzela-Ascoli theorem there exists a subsequence of $\left\{\varphi_{i}\right\}$ which converges in the $C^{2}$ topology to a nonnegative function $\varphi_{\infty} \in C^{2}(M)$ with $\left\|\varphi_{\infty}\right\|_{L^{p}\left(g_{\infty}\right)}=1$. Since the map $g \mapsto \lambda(g)$ is continuous with respect to the $C^{2}$ topology [BB], the function $\varphi_{\infty}$ satisfies (5.2) with $g=g_{\infty}$. By Theorem 4.1 in [LP], $\varphi_{\infty}$ is strictly positive and $C^{\infty}$.

Thus, after passing to a subsequence (and using the same index set) we may assume that $\left(\varphi_{i}\right)^{p-2} g_{i} \rightarrow\left(\varphi_{\infty}\right)^{p-2} g_{\infty}$. Now since $g_{\infty}$ is Einstein, it is the unique constant scalar curvature metric of volume 1 in its conformal class by Proposition 1.4 in [Sc2]. Hence $\left(\varphi_{\infty}\right)^{p-2} g_{\infty}=g_{\infty}$ and we see that $\varphi_{\infty} \equiv 1$.

Next, let $\mathcal{S}$ denote the set of unit volume metrics with constant scalar curvature. Since $g_{\infty}$ is not conformal to the round sphere metric, Theorem 4.44 in [Bes] asserts that the map

$$
\Phi: C^{\infty}(M) \times \mathcal{S} \rightarrow \mathcal{M} ;(f, g) \mapsto f \cdot g
$$

is a local ILH-diffeomorphism from a neighborhood of $\left(1, g_{\infty}\right)$ to $g_{\infty}$. Since $g_{i}$ and $\left(\varphi_{i}\right)^{p-2} g_{i} \in \mathcal{S}$, we certainly have $\left.\Phi\left(1,\left(\varphi_{i}\right)^{p-2} g_{i}\right)\right)=\Phi\left(\left(\varphi_{i}\right)^{p-2}, g_{i}\right)$, and hence $\varphi_{i} \equiv 1$ for all $i$ large. But $S_{g_{i}}>S_{g_{\infty}}$ and so $y(M)>S_{g_{\infty}}$.

To complete the proof, it remains to justify the uniform $C^{2, \beta}$ bound on the sequence $\left\{\varphi_{i}\right\}$. This follows in a straightforward manner from the proofs of Proposition 4.4 and Theorem 4.1 in [LP], together with the fact that, since $g_{i} \rightarrow g_{\infty}$ in $C^{3}$, all the constants in the Sobolev embedding and elliptic regularity theorems (Theorems $2.2,2.3,2.5$ ) in $[\mathrm{LP}]$ can be chosen to be independent of the individual metrics $g_{i}$.

More explicitly, since the conformal class $\left[g_{\infty}\right]$ is different from that of the round sphere, and $\varphi_{i}$ is a minimizer of the Yamabe problem for $\left[g_{i}\right]$, in applying the proof of Proposition 4.4 in [LP], we simply replace $\varphi_{s}$ there by $\varphi_{i}$ and replace Eq.(4.1) there by (5.2) above with $g=g_{i}$. As a result we get a bound for $\left\|\varphi_{i}\right\|_{L^{r}\left(g_{i}\right)}, r>p$, which is uniform in $i$ provided that the constant $C_{\epsilon}$ in Theorem 2.3 in [LP] can be chosen to be independent of $g_{i}$.

Next we apply the proof of Theorem 4.1 in [LP] with $s=p$, using again (5.2) with $g=g_{i}$ in place of Eq. (4.1) in [LP] and $\varphi_{i}$ in place of $\varphi$. The resulting $C^{2, \beta}$ bound is a continuous 
function of the constants coming from the embedding and elliptic regularity theorems used (cf. [GT], [Au2], [Heb]).

We can now apply Proposition 5.1 to the Einstein metrics resulting from the Graph Theorem. Recall that at the end of the proof of the Graph Theorem in section 3 we constructed a path of unit volume $G$-invariant metrics with positive scalar curvature tending to $+\infty$ at its two ends. Applying the gradient flow, which increases scalar curvature, we obtain a path which "remains hanging" on a $G$-invariant Einstein metric. If this Einstein metric $g$ is the only Einstein metric on this path, clearly one can produce a sequence $g_{i}$ satisfying the hypotheses of Proposition 5.1. Otherwise, one can choose a different Einstein metric lying on the same path as our $g_{\infty}$ with the same property. Hence we obtain

Corollary 5.3. Let $G / H$ be a compact connected homogeneous space. If the graph of $G / H$ has at least two non-toral components, then $G / H$ admits a homogeneous Einstein metric which does not realize the Yamabe invariant of $G / H$.

In [Koi] Koiso showed that an Einstein metric always has finite coindex in the space $\mathcal{S}$ of all constant scalar curvature metrics of unit volume on a closed manifold. He also computed the eigenvalues of the Hessian of the Hilbert action restricted to $\mathcal{S}$ in the case of the irreducible symmetric spaces of compact type. In many cases the symmetric metric is a strict local maximum while in other cases the Hessian has 0 as an eigenvalue. But for the symmetric spaces $S O(5), S p(n)$ and $S p(n) / U(n)$, there are positive eigenvalues, and since the eigenvectors of the Hessian (defined in terms of an elliptic operator) are always smooth, it follows that there exists a sequence of metrics as in Proposition 5.1. Therefore, the symmetric metrics on $S O(5), S p(n)$ and $S p(n) / U(n)$ do not realize the Yamabe invariant. When the Hessian of the symmetric metric has 0 eigenvalues but no positive eigenvalues, [Koi] is not sufficient to conclude whether a sequence of metrics as in Proposition 5.1 exists or not. But we will see in Example 6.7 that on one such symmetric space, namely $S U(2 n) / S p(n)$, such a sequence does exist and hence this metric does not realize the Yamabe invariant either. In [Je2] Jensen showed that on $S U(n)$ there exists a path of left-invariant metrics, containing the bi-invariant metric, with volume 1 and monotonically increasing scalar curvature. Hence the bi-invariant Einstein metric on $S U(n)$ is a degenerate critical point which again does not realize the Yamabe invariant.

\section{ExAmples AND Applications}

In this section we shall give many examples illustrating the use of the Graph Theorem. Let us recall that in order to show existence of a $G$-invariant Einstein metric on a compact homogeneous space $G / H$ with finite fundamental group it is enough to show that the graph has at least two components, if both $G$ and $H$ are connected, whereas in the general case we need show that the graph has at least two non-toral components (cf. Theorem 3.3 and Proposition 3.10).

We begin with a simple sufficient condition for the graph of $G / H$ to contain at least two components.

Proposition 6.1. Let $G$ be a compact Lie group, $H$ a closed subgroup such that $G / H$ is almost effective, and $K$ be an intermediate closed subgroup $(H \subset K \subset G)$. Assume that $\mathfrak{h}$ is a proper maximal $A d(H)$-invariant subalgebra of $\mathfrak{k}$, and $\mathfrak{k}$ is a proper maximal $A d(H)$-invariant subalgebra of $\mathfrak{g}$ satisfying $\mathfrak{z}(\mathfrak{k})=\mathfrak{c}\left(\mathfrak{k}_{s}\right)$. If $L$ is another closed intermediate subgroup such that $[\mathfrak{l}]$ is a vertex and $\mathfrak{l}$ is not conjugate to $\mathfrak{k}$ by an element of $N(H)^{0}$, then the graph of $G / H$ has at least two components. If $\mathfrak{g}$ is simple the assumption $\mathfrak{z}(\mathfrak{k})=\mathfrak{c}\left(\mathfrak{k}_{s}\right)$ is satisfied automatically. 
Proof. Using the bi-invariant metric $Q$, let $\mathfrak{g}=\mathfrak{k}_{s} \perp \mathfrak{m}$ and $\mathfrak{m}_{0}$ denote the subspace of $\mathfrak{m}$ which commutes with $\mathfrak{k}_{s}$. Consider $\mathfrak{k}_{s} \oplus \mathfrak{m}_{0}=\mathfrak{n}\left(\mathfrak{k}_{s}\right)$, which is $A d(H)$-invariant. Our assumptions and Proposition 4.2 imply that $\left[\mathfrak{n}\left(\mathfrak{k}_{s}\right)\right]=[\mathfrak{k}]=N(H)^{0} \cdot \mathfrak{k}$. Furthermore, $[\mathfrak{k}]$ is an isolated vertex by the maximality properties of $\mathfrak{h}$ and $\mathfrak{k}$. As well, by assumption $[\mathfrak{l}]$ is a vertex in the graph distinct from $[\mathfrak{k}]$ and the first part of the proposition is proved.

If $\mathfrak{g}$ is simple, then $\mathfrak{g}=\mathfrak{k}_{s} \oplus \mathfrak{m}_{0}$ would imply that $\mathfrak{m}_{0}=0$, which contradicts $\mathfrak{k} \neq \mathfrak{g}$.

Corollary 6.2. Let $G$ and $H$ be compact connected Lie groups such that $G / H$ is almost effective and $G$ is semisimple. Suppose that there exist a connected semisimple Lie group $K$, such that $\mathfrak{h}$ is maximal in $\mathfrak{k}$ and $\mathfrak{k}$ is maximal in $\mathfrak{g}$. If there exists a connected intermediate subgroup $L$ which is not isomorphic to $K$, then the graph of $G / H$ has at least two components.

Proof. If $\mathfrak{c}(\mathfrak{k})=0$, then Proposition 6.1 applies. If $\mathfrak{c}(\mathfrak{k}) \neq 0$ we conclude $\mathfrak{k} \oplus \mathfrak{c}(\mathfrak{k})=\mathfrak{g}$ since $\mathfrak{k}$ is maximal in $\mathfrak{g}$. By the same reason we get $\operatorname{dim} \mathfrak{c}(\mathfrak{k})=1$, that is $\mathfrak{g}=\mathfrak{k} \oplus \mathbb{R}$, which contradicts the assumption that $G$ is semisimple.

Now we can state our first class of examples. Let $G$ be a simple, compact, connected Lie group. Then Corollary 6.2 can be applied to the homogeneous spaces $(G \times G) / \Delta H$, where $H$ a maximal connected subgroup of $G$, and to $G / S^{1}$, where $S^{1} \subset S U(2) \subset G$ and $S U(2)$ is maximal in $G$. We set $K=\Delta G$ (respectively $K=S U(2))$ and $L=H \times H$ (respectively $L=T^{2}$ ). Further, a very large class of examples can be obtained as follows. Let $H \subset K \subset G$ where $H$ is a simple compact connected Lie group and $K$ and $G$ are simple, compact, classical Lie groups. Assume that the inclusions $H \subset K$ and $K \subset G$ are respectively given by irreducible representations $\rho$ and $\sigma$. Dynkin showed ([Dy], Theorem 1.5) that apart from a few exceptional representations $\rho$ and $\sigma, H$ will be a maximal subgroup of $K$ and $K$ will be a maximal subgroup of $G$. He also showed that apart from a few representations $\rho$ and $\sigma, \rho \circ \sigma$ is reducible (cf. [WZ1]). In most cases we can then write $\rho \circ \sigma=\rho_{1} \oplus \rho_{2}$, with $H \subset K^{\prime}=K_{1} \times K_{2}$, where $K_{1}$ and $K_{2}$ are classical simple Lie groups with $\rho_{i}(H) \subset K_{i}$. Corollary 6.2 then implies that the graph of $G / H$ has more than one component. As a concrete example, we have:

Example 6.3. Let $G=S O\left(2 n^{2}+n\right), n \geq 2$, and $H=S O(3)$, where $H$ is embedded into $K=S p(n) / \mathbb{Z}_{2}$ via the irreducible $2 n$-dimensional unitary representation of $S U(2)$, which is symplectic, and $K$ is embedded into $G$ by the adjoint representation. Up to conjugacy, $H$ is the principal 3-dimensional subgroup of $K$. By using Table I of [Dy] we see that $H$ is maximal in $K$, which is in turn maximal in $G$. On the other hand, the representation corresponding to the inclusion of $H$ in $G$ is the second symmetric power $S^{2}((2 n-1))$ of $(2 n-1)$, which denotes the irreducible unitary representation of $S U(2)$ with dimension $2 n$. Since

$$
S^{2}((2 n-1))=(4 n-2) \oplus \cdots \oplus(2),
$$

it follows that $H$ lies in some $L$ of the form $S O(3) \times S O\left(2 n^{2}+n-3\right)$ which is not isomorphic to $K$. Therefore the graph of $G / H$ has at least two components.

It is interesting to note that the isotropy representation of $G / H$ is quite complicated. It is actually given by

$$
\begin{aligned}
& \Lambda^{2}\left(S^{2}((2 n-1))\right)-(2)=((8 n-6) \oplus \cdots \oplus(2)) \oplus \cdots \oplus((10) \oplus(6) \oplus(2)) \\
& \oplus \bigoplus_{1 \leq i<j \leq n}((4(i+j-1)) \oplus \cdots \oplus(4(j-i))) .
\end{aligned}
$$

There are repeated irreducible summands, e.g., (2) occurs $n-1$ times, and the total number of summands equals $\left(4 n^{2}+7 n+6\right)(n-1) / 6$. The set $\mathcal{M}_{1}^{G}$ is hence quite complicated and its 
dimension is arbitrarily large so that explicit calculations seem to become impossible. There are no trivial summands, and so $N(H)^{0}=H$.

Interestingly, the graph of $G / H$ contains a third vertex when $n>3$. To see this, consider the inclusions $S O(3) \subset S O(2 n+1) \subset S O\left(2 n^{2}+n\right)$, where the first inclusion is given by the irreducible orthogonal representation of $S O(3)$ of dimension $2 n+1$, and the second inclusion by the adjoint representation of $S O(2 n+1)$. The composition of the two inclusions is given by the representation $\Lambda^{2}((2 n))=(4 n-2) \oplus \cdots \oplus(2)$, which is the same embedding as (6.4). So we obtain the same homogeneous space. When $n \neq 3$, then $S O(3)$ is maximal in $S O(2 n+1)$, by Dynkin, while for $n=3$ we have $S O(3) \subset G_{2} \subset S O(7)$. Since $S O(2 n+1)$ is always maximal in $S O\left(2 n^{2}+n\right)$ (the quotient is isotropy irreducible), and not isomorphic to $S p(n) / \mathbb{Z}_{2}$ if $n \neq 2$, Proposition 6.1 shows that $[\mathfrak{s o}(n+1)]$ is another isolated vertex in the graph when $n>3$.

Example 6.5. Our next example shows that it may be advantageous to choose a group $G$ which is not connected. This is why we have been careful not to make the assumption in any of our theorems that $G$ is connected, unless it was necessary. Let $G_{0}=S O(k p+k q)$, $H_{0}=S O(p)^{k} \times S O(q)^{k}, p, q \geq 3, k \geq 2$, and let $\Gamma$ be the symmetric group $\mathrm{S}_{k}$ on $k$ objects. $\Gamma$ acts on $S O(k p)$ by automorphisms interchanging the simple factors of $S O(p)^{k}$. Hence $\Gamma$ acts by automorphisms "diagonally" on $G_{0}$ interchanging the simple factors of $S O(p)^{k}$ and $S O(q)^{k}$. Let $G=S O(k p+k q) \ltimes \Gamma$ and $H=\left(S O(p)^{k} \times S O(q)^{k}\right) \ltimes \Gamma$. Then $G / H=G_{0} / H_{0}$ as manifolds.

The irreducible summands of the isotropy representation of $G / H$ are given by

$$
\begin{aligned}
\mathfrak{m}_{1} & =\left\{\rho_{i} \otimes \rho_{j} \mid i \neq j, 1 \leq i, j \leq k\right\} \\
\mathfrak{m}_{2} & =\left\{\mu_{i} \otimes \mu_{j} \mid i \neq j, 1 \leq i, j \leq k\right\} \\
\mathfrak{m}_{3} & =\left\{\rho_{i} \otimes \mu_{i} \mid 1 \leq i \leq k\right\} \\
\mathfrak{m}_{4} & =\left\{\rho_{i} \otimes \mu_{j} \mid i \neq j, 1 \leq i, j \leq k\right\}
\end{aligned}
$$

where $\rho_{i}$ is the standard representation of the $i$ th copy of $S O(p)$ and $\mu_{i}$ is the standard representation of the $j$ th copy of $S O(q)$.

The summands $\mathfrak{m}_{1}$ and $\mathfrak{m}_{2}$ give rise to the vertices $[k s o(p) \oplus s o(k q)]$ and $[s o(k p) \oplus k s o(q)]$ which are joined to $[s o(k p) \oplus s o(k q)]$. The summand $\mathfrak{m}_{3}$ corresponds to a second component given by the isolated vertex $[k s o(p+q)]$. Hence the graph of $G / H$ has at least two non-toral components. (If $k=2$, there exists a third component.)

Note though that the graph of $G_{0} / H_{0}$ is connected. In general, $\mathcal{M}_{1}^{G}$ is a subset of $\mathcal{M}_{1}^{G_{0}}$ which is invariant under the Ricci flow. But the graph of $G_{0} / H_{0}$ can be quite different from the graph of $G / H$, and hence the global behavior of $S$ on $\mathcal{M}_{1}^{G}$ and $\mathcal{M}_{1}^{G_{0}}$ are not the same, as the example shows.

In contrast to the previous examples, in the remaining 5 the algebraic Einstein equations can be solved explicitly. The main point we want to make in these examples is that they illustrate quite interesting phenomena for the general behavior of $S$. The first two examples concern Einstein metrics on irreducible symmetric spaces of compact type. Of course if $(G, H)$ is a symmetric pair, then $G / H$ is isotropy irreducible and hence $\mathcal{M}_{1}^{G}$ consists of a single point which is Einstein. But there are some examples where a subgroup $G^{*}$ of $G$ still acts transitively. In such cases, the $G^{*}$-invariant metrics may depend on several parameters, and therefore there could be further $G^{*}$-invariant Einstein metrics. For rank one symmetric spaces, this was examined completely in $[\mathrm{Zi}]$. For higher rank irreducible symmetric spaces not isometric to a compact simple Lie group with a bi-invariant metric, this was examined in [Ke], and we will study two of her examples from the present viewpoint. Before doing so, let us point out that by comparing the Einstein constants of the explicit solutions in $[\mathrm{Zi}]$ and $[\mathrm{Ke}]$, one can show that the 
symmetric Einstein metric is always the one with largest scalar curvature in $\mathcal{M}_{1}^{G}$, i.e., the "best" homogeneous Einstein metric on these spaces. But this only follows by an explicit computation in each case, and it would be interesting to obtain a conceptual understanding of this fact. In particular it is not known if the above fact remains true in the only case not studied in [Zi] and $[\mathrm{Ke}]$, i.e., left-invariant metrics on a compact simple Lie group. Here the isometry group of the bi-invariant symmetric metric is $G \times G$, and there are many subgroups acting transitively, e.g., $G \times K$ for any $K$. This situation has been studied extensively in [DZ], giving rise to many left-invariant Einstein metrics.

Example 6.6. Our first example is the symmetric space $G_{2}^{+}\left(\mathbb{R}^{7}\right)$ of oriented 2-planes in $\mathbb{R}^{7}$. Since $G_{2} \subset S O(7)$ acts transitively on this space with isotropy group $U(2)$ we have $G / H=$ $G_{2} / U(2)=G_{2}^{+}\left(\mathbb{R}^{7}\right)$ as manifolds. The intermediate subalgebras are $S U(3)$ and $S O(4)$ and hence the graph consists of two isolated vertices. The $G$-invariant metrics depend on three parameters and the scalar curvature is given by:

$$
S(a, b, c)=\frac{16}{3 a}+\frac{16}{b}+\frac{16}{c}-\frac{4 a}{3 b^{2}}-\frac{2 a}{b c}-\frac{2 b}{a c}-\frac{2 c}{a b} .
$$

In $[\mathrm{Ke}]$ the critical points, subject to the volume constraint $a^{2} b^{4} c^{4}=1$, were determined.
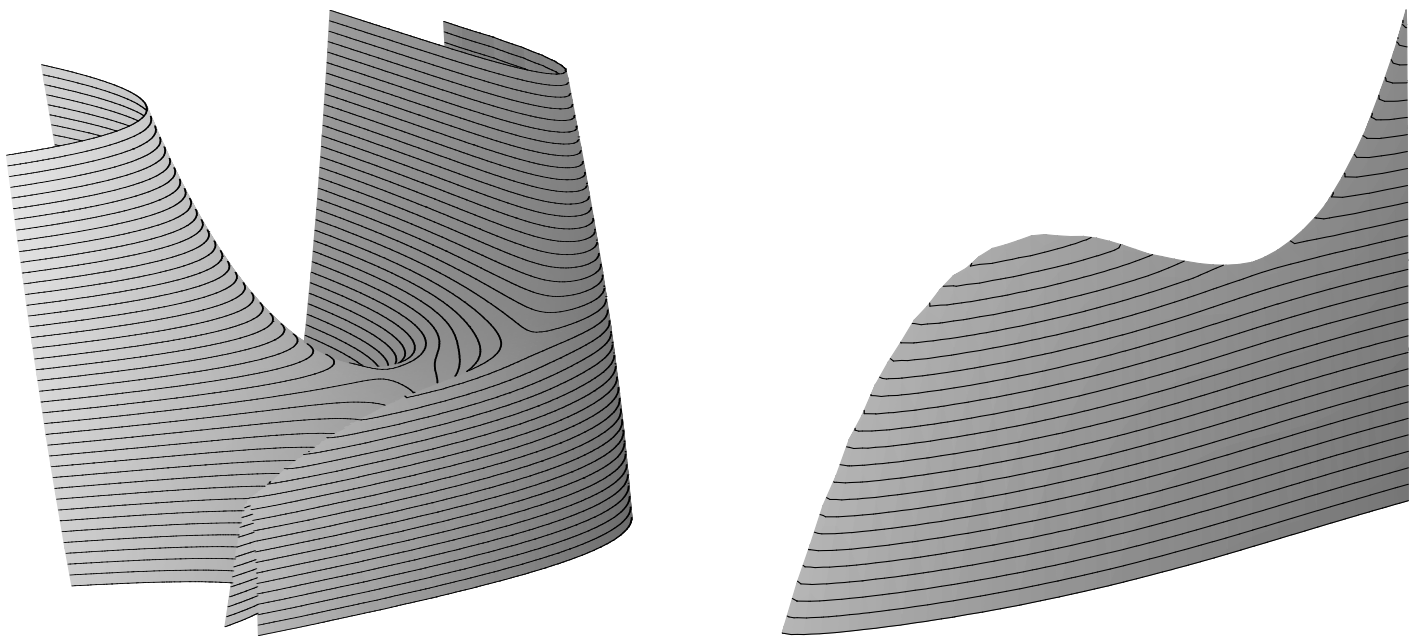

Figure 2. Einstein metrics on $G_{2} / U(2)=G_{2}^{+}\left(\mathbb{R}^{7}\right)$.

There are 3 critical points, one of which is the symmetric metric. The global behavior of $S$ subject to $v o l=a^{2} b^{4} c^{4}=1$ is depicted in Figure 2. In the first picture, one clearly sees the two subgroup directions where the scalar curvature goes to $+\infty$ and the critical point of coindex one which has to exist for global variational reasons. The two other critical points, including the symmetric metric, are located on the ridge in the front of the picture, which are made visible in the second picture where the symmetric metric is a local max and the third critical point is another saddle point. Notice that a small deformation could destroy the symmetric metric and the second saddle point. Explicit computations show that the symmetric metric has the largest scalar curvature $[\mathrm{Ke}]$. It is not known if this metric realizes the Yamabe invariant or not.

One obtains almost identical pictures and the same general conclusions on the symmetric space $G_{3}\left(\mathbb{R}^{8}\right)$ on which $\operatorname{Spin}(7)$ acts transitively and hence $G_{3}\left(\mathbb{R}^{8}\right)=\operatorname{Spin}(7) / S O(4)$. 
Example 6.7. Our next example is the compact symmetric space $M=S U(2 n) / S p(n), n>2$. We will show that the symmetric metric does not realize the Yamabe invariant. Here $S U(2 n-1)$ $\subset S U(2 n)$ acts transitively on $S U(2 n) / S p(n)$ with isotropy group $S p(n-1)$ and hence $M=$ $S U(2 n-1) / S p(n-1)=G^{\prime} / H^{\prime}$ as manifolds. The only intermediate subgroups are $S p(n-1) \cdot S^{1}$ and $S U(2 n-2) \subset U(2 n-2)$. Hence the graph is connected. The set of $S U(2 n-1)$-invariant metrics on $M$ depends on 3 parameters and the scalar curvature is given by

$$
S(a, b, c)=(2 n-1)\left(\frac{4(n-1)(n-2)}{a}+\frac{8(n-1)}{b}-\frac{c}{b^{2}}-\frac{(n-2) a}{b^{2}}\right) .
$$

Kerr showed that the only critical point of $S$, subject to the constraint $a^{(2 n-1)(n-2)} b^{4(n-1)} c=1$ on the volume, is the symmetric metric $g_{0}$ itself, given by $b=\frac{1}{2} a, c=\frac{n}{2 n-1} a$.

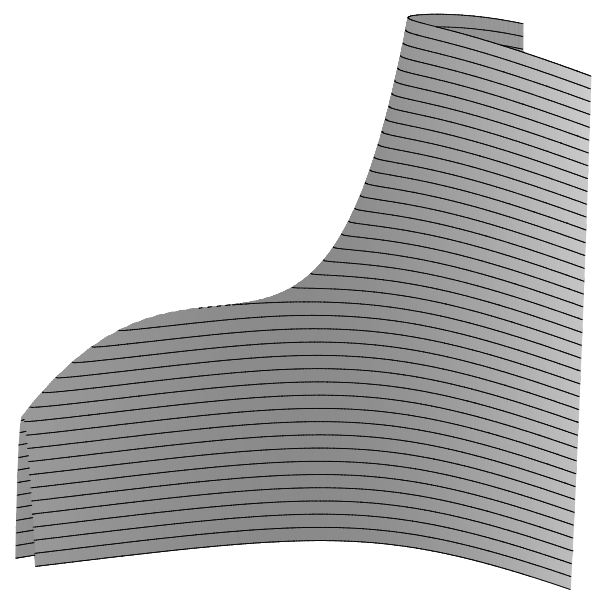

Figure 3. Einstein metrics on $S U(2 n) / S p(n)$.

One can easily show via explicit computations that the Hessian of $S$ at $g_{0}$ has a negative and a 0 eigenvalue. But we want to make the stronger conclusion that $g_{0}$ is not a local maximum of $S$ on $\mathcal{M}_{1}^{G^{\prime}}$. Proposition 5.1 then implies that $g_{0}$ does not realize the Yamabe invariant. To this end, suppose the contrary. Since $g_{0}$ is the unique critical point of $S$, there exists $\epsilon>0$ such that for all $g \in B_{\epsilon}\left(g_{0}\right) \backslash\left\{g_{0}\right\} \subset \mathcal{M}_{1}^{G^{\prime}}$ we have $S(g)<S\left(g_{0}\right)=S_{0}$. Hence there exists $\delta>0$, such that a connected component of $S^{-1}\left(S_{0}-\delta\right)$ is contained in $B_{\epsilon}\left(g_{0}\right)$. Next we let $\gamma_{1}$ be a straight line in $\mathcal{M}^{G^{\prime}}$ joining $g_{0}$ and a normal homogeneous metric $g_{1}$ and one easily checks, using the explicit formula for the scalar curvature, that along this path $S>0$. Now note that $S U(2 n-2)$ is a non-toral subalgebra of $G^{\prime}$ containing $H^{\prime}$ properly. As in the proof of the Graph Theorem, it follows that there exists a path $\gamma_{2}$ in $\{S>0\}$ joining $g_{1}$ and high energy levels of $S$. We conclude that the level set $S^{-1}\left(S_{0}-\delta\right)$ is disconnected and that there exist two path components of it which can be joined by a path in $\{S>0\}$. As in the proof of the Graph Theorem the existence of a critical point $g_{0}^{\prime}$ in $\left\{S \leq S_{0}-\delta\right\}$ follows. Thus $g_{0} \neq g_{0}^{\prime}$, a contradiction.

In Figure 3 one can see (in the case of $n=3$ ) the degeneracy of the symmetric metric as a critical point. The fact that $g_{0}$ is not a local max is barely visible. Clearly a small deformation of the function $S$ could destroy this critical point and hence there is no reason for its global existence.

Example 6.8. An interesting non-symmetric example is $G / H=\left(S^{3} \times S^{3}\right) / S_{r}^{1}$ where $S_{r}^{1}=$ $\left(e^{i p t}, e^{i q t}\right)$ with $p, q$ relatively prime integers and $r=p / q$. By using the interchange of the two factors in $S^{3} \times S^{3}$ and conjugation in each factor, we can assume that $0 \leq p \leq q$ and hence 
$0 \leq r \leq 1$. The value $r=0$, i.e., $p=0, q=1$, represents the product homogeneous space $S^{3} \times S^{2}$ and $r=1$ the Stiefel manifold $V_{2}\left(\mathbb{R}^{4}\right)=S O(4) / S O(2)=\left(S^{3} \times S^{3}\right) / \Delta S^{1}$. Recall that for all $r$, the manifolds $\left(S^{3} \times S^{3}\right) / S_{r}^{1}$ are diffeomorphic to $S^{3} \times S^{2}$ [WZ2]. From the classification of homogeneous 5-manifolds, it follows that a homogeneous metric on $S^{3} \times S^{2}$ must be a $G$ invariant metric as above, for some value of $r$. Hence we will be able to examine the set of all homogeneous Einstein metrics completely in a non-trivial example which has infinitely many distinct transitive actions (by $S^{3} \times S^{3}$ ).

The case $r=0$ is very special since in this case every homogeneous metric consists of a product metric of an arbitrary left-invariant metric on $S^{3}$ and a round sphere metric on $S^{2}$ and hence the space of homogeneous metrics is 7-dimensional. This space also contains as proper subspaces the set of $S O(4) \times S O(3)$-invariant metrics and the set of $U(2) \times S O(3)$-invariant metrics. Jensen showed in [Je2] that the bi-invariant metric is a global maximum for the scalar curvature functional on the set of volume 1 left-invariant metrics on $S^{3}$. Hence the product Einstein metric on $S^{3} \times S^{2}$, which is clearly the only critical point, has coindex 1 .

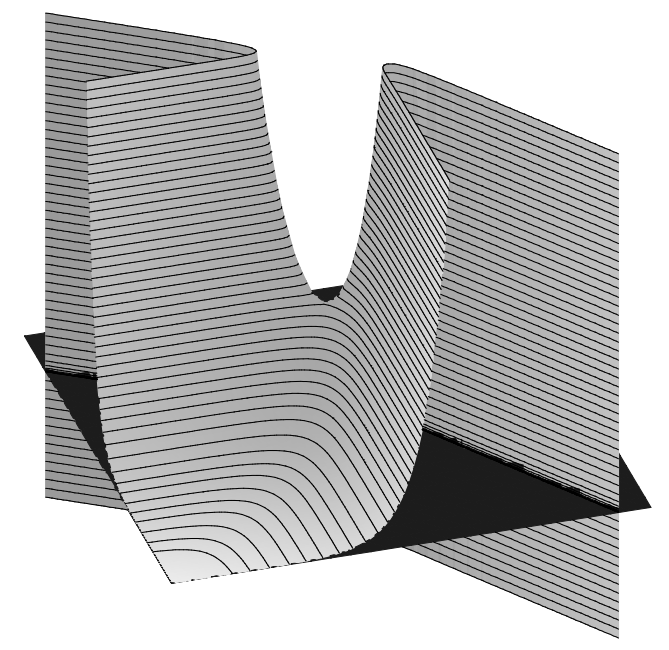

Figure 4. Einstein metrics on $\left(S^{3} \times S^{3}\right) / S_{r}^{1}$.

We will now study the more interesting case $r \neq 0,1$, which should be viewed as being typical for a homogeneous space $G / T$ where $T$ is a generic non-maximal torus. The scalar curvature on the set of all $G$-invariant metrics is equal to (see e.g. [Rod]):

$$
S(a, b, c)=\frac{8}{b}+\frac{8}{c}-\frac{2 a}{\left(1+r^{2}\right) b^{2}}-\frac{2 a r^{2}}{\left(1+r^{2}\right) c^{2}},
$$

subject to $v o l=a b^{2} c^{2}=1$. These metrics can be described geometrically as scaling separately, with respect to the fibration $S^{1} \rightarrow\left(S^{3} \times S^{3}\right) / S_{r}^{1} \rightarrow\left(S^{3} \times S^{3}\right) / T^{2}=S^{2} \times S^{2}$, the metric on the fibre by $a$ and on each round 2 -sphere in the base by $b$ and $c$ respectively. This describes all $G$-invariant metrics on $G / H$ and one shows that $S$ has a unique critical point (cf. [WZ3]).

The graph consists of the vertices $T^{2}$, connected to $S^{3} \times S^{1}$ and $S^{1} \times S^{3}$ and hence the graph is connected. A picture of $S$ subject to the volume constraint $a b^{2} c^{2}=1$ is shown in Figure 4 .

The first interesting feature of this picture is that one clearly sees the 0-Palais-Smale sequences (notice that the plane $S=0$ is included in the same picture), corresponding to the $T^{2}$ vertex. The picture also suggests that, although the graph is connected, the unique critical point should exist for global reasons. One can define a reduced graph of $G / H$ where in the graph of $G / H$ 
one removes the vertices corresponding to toral subgroups. The picture then suggests that $S$ has a critical point if the reduced graph has at least two components.

If one solves the Einstein equations explicitly as in [Rod], one can easily draw the graph of the Einstein metrics $(a, b, c)$ and the scalar curvature as a function of $r$ as in Figure 5.
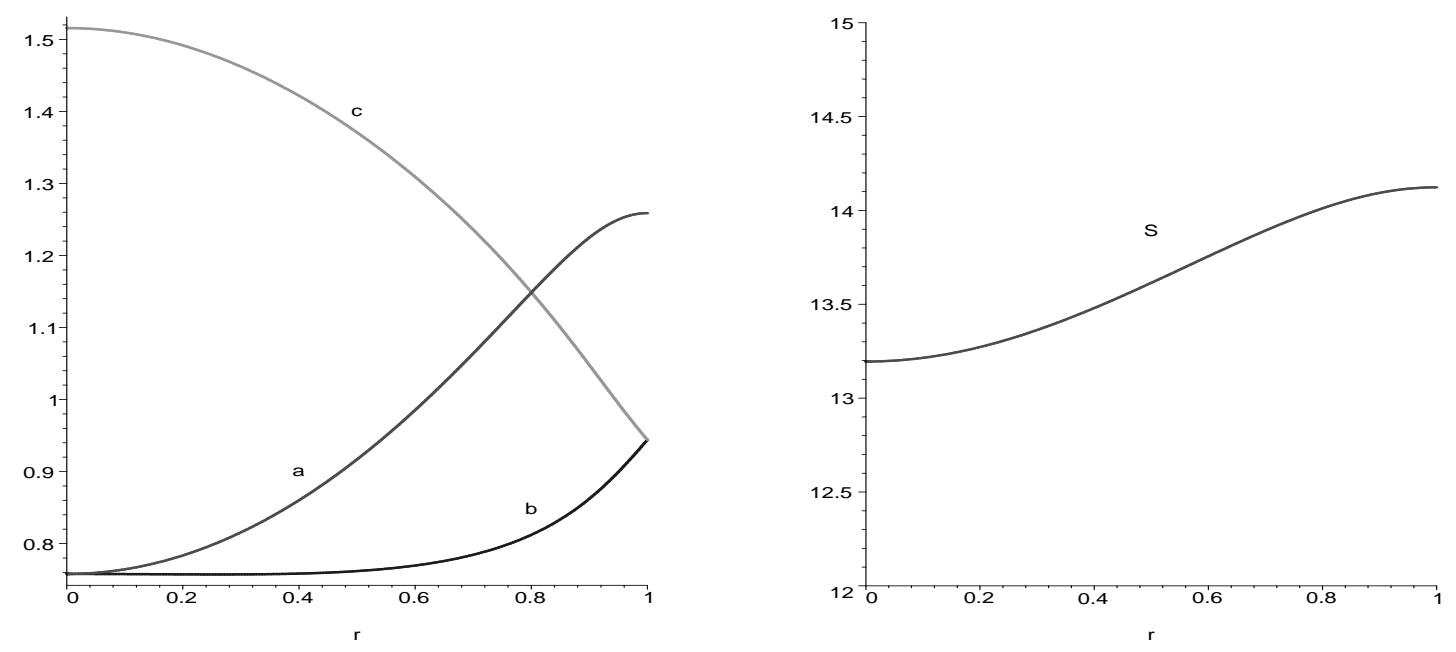

FiguRE 5. Metric and scalar curvature of the Einstein metric on $S^{3} \times S^{3} / S_{r}^{1}$.

The Einstein metric is smooth in $r$, exists locally, and is real analytic for all $r$. For $r$ rational it extends (uniquely) to a homogeneous Einstein metric on $S^{3} \times S^{2}$ and for $r$ irrational it extends to the non Hausdorff space $\left(S^{3} \times S^{3}\right) / \mathbb{R}$.

But notice that the picture in Figure 5 is misleading (and indeed seems to contradict our finiteness result (1.9)) since the normalization $v o l=a b^{2} c^{2}=1$ only makes sense for a fixed value of $r$. In principle one also needs to multiply $a b^{2} c^{2}$ by the volume of the metric induced

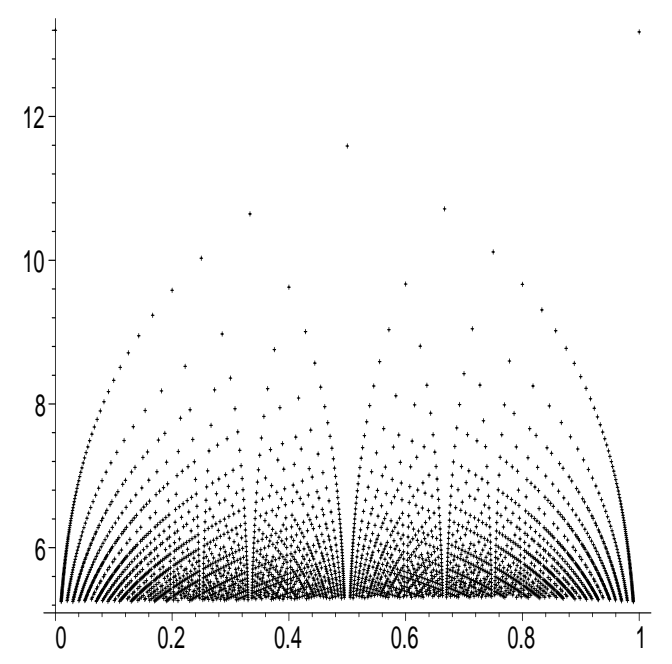

Figure 6. Normalized scalar curvature of the Einstein metrics on $S^{2} \times S^{3}$.

on $\left(S^{3} \times S^{3}\right) / S_{r}^{1}$ by the bi-invariant metric on $S^{3}(1) \times S^{3}(1)$, which depends on $r$. Hence a correct normalization is $v o l=a b^{2} c^{2} / \sqrt{p^{2}+q^{2}}=1$. Instead of $S(a, b, c)$ we should therefore 
study $S(a, b, c) / \sqrt[10]{p^{2}+q^{2}}$. The graph of the corrected scalar curvature of the Einstein metrics at many rational points $r$ is represented in Figure 6, and shows that the two metrics competing for the largest scalar curvature are the product metric $(r=0)$ and the Jensen metric $(r=1)$. A computation shows that the product Einstein metric has scalar curvature $S=13.195$ and the Jensen metric $S=13.177$. Hence the product metric on $S^{3} \times S^{2}$ is the one with largest scalar curvature, i.e., the best homogeneous Einstein metric. In the next example we will see a conceptual reason for the product metric to have a larger scalar curvature. It again follows that the best homogeneous Einstein metric does not realize the Yamabe invariant.

Example 6.9. We now consider the previous example in the special case of $r=1$, i.e., $V_{2}\left(\mathbb{R}^{4}\right)=\left(S^{3} \times S^{3}\right) / \Delta S^{1}$. This example should be viewed as being typical for a space $G / T$ where $T$ is a non-maximal torus which is not generic.

The example is special since the two non-trivial summands in the isotropy representation are equivalent, giving rise to a 5 -dimensional family of invariant metrics or a 4 -dimensional $\mathcal{M}_{1}^{G}$. The scalar curvature of such a metric with respect to the natural coordinates $(a, b, c, d, e)$, where $a, b, c$ are the diagonal entries of the metric and $d, e$ the off-diagonal ones, is given by:

$$
S(a, b, c, d, e)=8\left(\frac{1}{a}+\frac{c+b}{\Delta}-\frac{b c}{a \Delta}-\frac{a(b-c)^{2}}{8 \Delta^{2}}-\frac{a}{4 \Delta}\right)
$$

with $v o l=a \Delta^{2}=1$ and $\Delta=b c-d^{2}-e^{2}$.

The special 3-dimensional subfamily $d=e=0$ of diagonal metrics is invariant under the Ricci flow since it is the fixed point set of $S O(2)=N(H) / H$ in $\mathcal{M}_{1}^{G}$. For these metrics $S$ agrees with the scalar curvature in the previous example and hence one again gets a unique critical point in this subfamily by [WZ3]. This metric is called the Jensen metric on $V_{2}\left(\mathbb{R}^{4}\right)$ [Je3], which is given by $a=\frac{4}{3} b=\frac{4}{3} c, d=e=0$. It is not only invariant under the gauge group $N(H) / H$, but under the slightly larger group $\operatorname{Aut}(G, H)$ which consists of all automorphisms of $G$ which preserve $H$. In our case $A u t(G, H)=O(2)$.

As was shown in $[\mathrm{ADF}]$ there exist further critical points among the non-diagonal metrics. They are all isometric to each other and consist of the two critical circles

$$
\begin{aligned}
& b=\frac{3}{2} a, c=\frac{1}{2} a, d=\frac{a}{2} \cos (\theta), e=\frac{a}{2} \sin (\theta) \\
& b=\frac{1}{2} a, c=\frac{3}{2} a, d=\frac{a}{2} \cos (\theta), e=\frac{a}{2} \sin (\theta) .
\end{aligned}
$$

Observe though that these metrics simply consist of an $\operatorname{Aut}(G, H)$ orbit of the product Einstein metric on $S^{3} \times S^{2}$. Indeed, the diffeomorphism $f:\left(S^{3} \times S^{3}\right) / \Delta S^{1} \rightarrow S^{3} \times S^{2}$ defined by $f\left((x, y) S^{1}\right)=\left(x y^{-1}, x S^{1}\right)$ pulls back the product Einstein metric to an $\left(S^{3} \times S^{3}\right)$-invariant metric which is given by $b=\frac{3}{2} a, c=\frac{1}{2} a, d=-\frac{1}{2} a, e=0$.

A calculation shows that the Jensen metric is a critical point with coindex 3 and index 1 and the product metric has coindex 1 , index 2 and a 0 eigenvalue corresponding to the direction tangent to the critical circle. Hence in this case $S$ is a Morse-Bott function.

For the graph we have, in addition to the subgroups already considered in Example 6.9, another intermediate subgroup $\Delta S^{3}$ which produces a second component in the graph. Hence in this case the graph theorem produces the product metric instead of the Jensen metric. Notice that Morse theory now forces the scalar curvature of the product metric to be the largest. Indeed if we let $r \rightarrow 1$ in Example (6.9), we obtain a one-dimensional path which remains hanging on the unique critical point for $r \neq 1$. This path for $r=1$ now sits on a critical point of coindex 3 and hence can be pushed up further by the gradient flow and must then remain hanging on 
a new critical point of coindex 1 . This critical point is the product metric since there are no further critical points.

Example 6.10. Our final example shows that the existence and non-existence of Einstein metrics is in some cases due to a mere coincidence, i.e. it seems unlikely that for such Einstein metrics a global reason for its existence can be found.

The first space is $S O(9) /(S O(3) U(1)) S O(3)$ which has no invariant Einstein metrics and the second space is $S O(10) /(S O(3) U(1)) S O(4)$ which carries two invariant Einstein metrics. In both
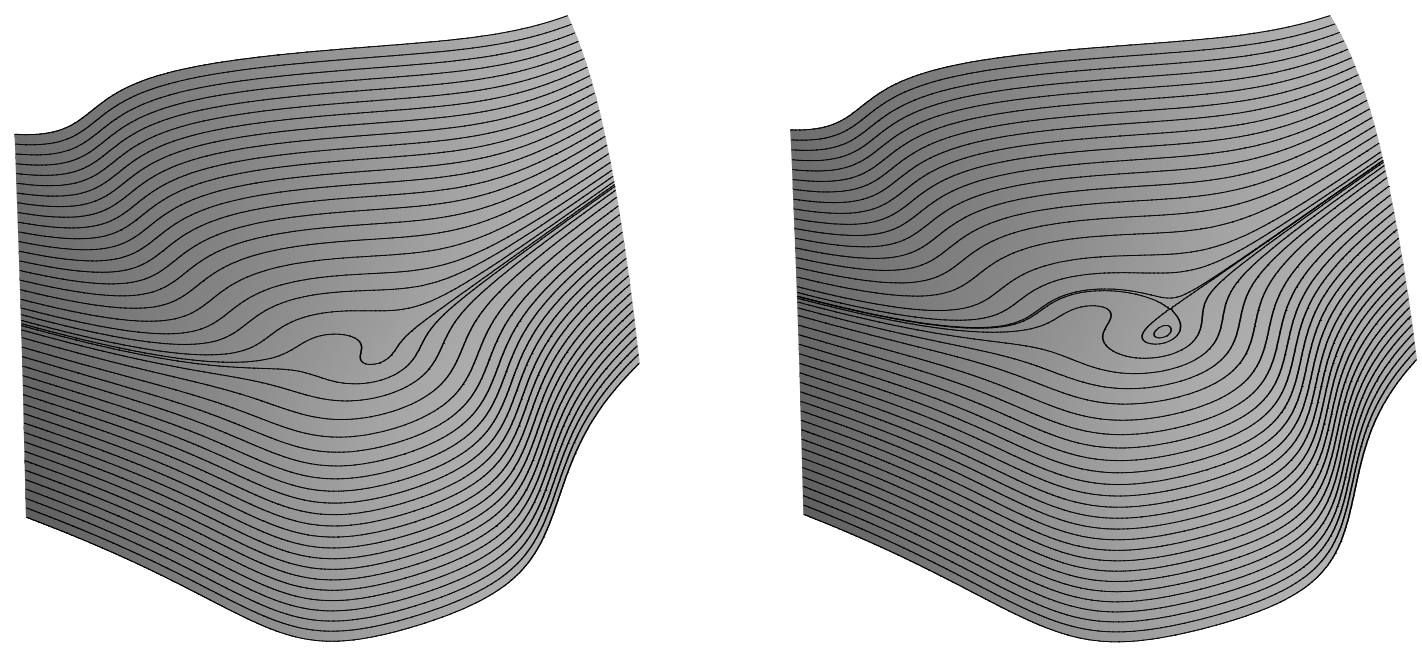

Figure 7. Non-existence on $G / H=S O(9) /(S O(3) U(1)) S O(3)$ and existence on $G / H=S O(10) /(S O(3) U(1)) S O(4)$.

cases the graph is connected. The embedding of the subgroup $H$ is given by $S O(3) U(1) S O(k) \subset$ $U(3) S O(k) \subset S O(6) S O(k)$ for $k=3,4$. This family of spaces has been investigated in [PS] (for all $k$ ), where non-existence (resp. existence) of homogeneous Einstein metrics has been proved. The scalar curvature function when $k=3$ (resp. 4) is given by

$$
\begin{aligned}
& S(a, b, c)=\frac{15}{14 a}+\frac{12}{7 b}+\frac{9}{c}-\frac{15 a}{28 c^{2}}-\frac{9 b}{14 c^{2}}-\frac{5 a}{28 b^{2}}, \\
& S(a, b, c)=\frac{15}{16 a}+\frac{3}{2 b}+\frac{12}{c}-\frac{5 a}{8 c^{2}}-\frac{3 b}{4 c^{2}}-\frac{5 a}{32 b^{2}} .
\end{aligned}
$$

The graph of $S$ subject to the volume condition $a^{5} b^{6} c^{18}=1$ (respectively $a^{5} b^{6} c^{24}=1$ ) is depicted in Figure 7. These two pictures clearly indicate that the global behavior of the scalar curvature function is the same. But when passing from the left picture to the right one, a (local) bifurcation occurs.

\section{REFERENCES}

[ADF] D. V. Alekseevsky, I. Dotti and C. Ferraris, Homogeneous Ricci positive 5-manifolds, Pacific J. Math. 175 (1996), 1-12.

[AK] D. V. Alekseevsky and B. N. Kimel'fel'd, Structure of homogeneous Riemann spaces with zero Ricci curvature, Func. Anal. Appl. 9 (1975), 97-102.

[An] M. T. Anderson, Convergence and rigidity of manifolds under Ricci curvature bounds, Invent. Math. 102 (1990), 429-445.

[Ar] A. Arvanitoyeorgos, New invariant Einstein metrics on generalized flag manifolds, Trans. A.M.S. 337 (1993), 981-995. 
[Au1] T. Aubin, Équations différentielle non linéaire it problème de Yamabe concernant la courbure scalaire. J. Math. Pures Appl. 55 (1976), 269-296.

[Au2] T. Aubin, Nonlinear analysis on manifolds. Monge-Ampère equations, Springer-Verlag (1982).

[B] M. Berger, Quelques formules de variation pour une structure riemannienne, Ann. scient. Éc. Norm. Sup. (4 $4^{e}$-série) t. 3 (1970), 285-294.

[BB] L. Bérard Bergery, Scalar curvature and isometry groups, in "Spectra of Riemannian manifolds", ed. M. Berger, S. Murakami, T. Ochiai, Kagai, Tokyo (1983), 9-28.

[Bes] A. Besse, Einstein Manifolds, Ergeb. Math. Grenzgeb., 3. Folge, Band 10, Springer-Verlag, (1987).

[BCR] J. Bochnak, M. Coste and M.-F. Roy, Real algebraic geometry, Ergeb. Math. Grenzgeb., 3. Folge, Band 36, Springer-Verlag, (1998).

[Bo] S. Bochner, Curvature and Betti numbers, Ann. of Math. 49 (1948), 379-390.

[Bö1] C. Böhm, Inhomogeneous Einstein metrics on low-dimensional spheres and other low-dimensional spaces, Invent. Math. 134 (1998), 145-176.

[Bö2] C. Böhm, The scalar curvature of homogeneous metrics, preprint (2002).

[BtD] T. Bröcker and T. tom Dieck, Representations of compact Lie groups, Springer-Verlag, (1985).

[CC] J. Cheeger and T. H. Colding, On the structure of spaces with Ricci curvature bounded below I, J. Differ. Geom. 46 (1997), 406-480.

[Dy] E. B. Dynkin, Maximal subalgebras of the classical groups, Transl. Am. Math. Soc. Series 2, Vol. 6 (1957), 245-378.

[DZ] J.E.D'Atri and W.Ziller, Naturally reductive metrics and Einstein metrics on compact Lie groups, Memoirs Amer. Math. Soc. 18, No. 215 (1979).

[Es] J.-H. Eschenburg, Lecture notes on symmetric spaces, preprint 1997.

[Fu] K. Fukaya, Hausdorff convergence of Riemannian manifolds and its applications, Advanced Studies in Pure Mathematics 18-I, Recent Topics in Differential and Analytic Geometry (1990), 143-238.

[GT] D. Gilbarg, N. Trudinger, Elliptic partial differential equations of second order, 2nd edition, SpringerVerlag (1998).

[Go] M. Golubitsky, Primitive actions and maximal subgroups of Lie groups, J. Diff. Geom. 7 (1972), $175-191$.

[Gr] M. Gromov, Groups of polynomial growth and expanding maps, Publ. Math. IHES 53 (1981), 53-73.

[Heb] E. Hebey, Sobolov spaces on Riemannian manifolds, Lecture Notes in Math. 1635, Springer-Verlag (1996).

[He] S. Helgason, Differential geometry, Lie groups, and symmetric spaces, Academic Press New-York and London, second edition, (1978).

[Hi] D. Hilbert, Die Grundlagen der Physik, Nachr. Akad. Wiss. Gött. (1915), 395-407.

[Je1] G. Jensen, Homogeneous Einstein spaces of dimension 4, J. Diff. Geom. 3 (1969), 309-349.

[Je2] G. Jensen, The scalar curvature of left-invariant Riemannian metrics, Indiana U. Math. J. 20 (1971), 1125-1144.

[Je3] G. Jensen, Einstein metrics on principal fibre bundles, J. Diff. Geom. 8 (1973), 599-614.

[Ke] M. Kerr, Some new homogeneous Einstein metrics on symmetric spaces, Trans. Amer. Math. Soc. 348 (1996), 153-171.

[Ki] M. Kimura, Homogeneous Einstein metrics on certain Kähler C-spaces, Advanced Studies in Pure Mathematics 18-I, Recent Topics in Differential and Analytic Geometry (1990), 303-320.

[Ko] O. Kobayashi, Scalar curvature of a metric with unit volume, Math. Ann. 279 (1987), 253-265.

[Kob] S. Kobayashi, Topology of positively pinched Kähler manifolds, Tôhoku Math. J. 15 (1963), $121-139$.

[Koi] N. Koiso, Rigidity and stability of Einstein metrics-the case of compact symmetric spaces, Osaka J. Math. 17 (1980), 51-73.

[LP] J. M. Lee and T. H. Parker, The Yamabe problem, Bull. Amer. Math. Soc. (N.S.) 17 (1987), 37-91.

[MZ] D. Montgomery and L. Zippin, Topological transformation groups, Wiley, (1955), reprinted by Krieger (1974).

[Ni] Y. G. Nikonorov, Compact homogeneous Einstein 7-manifolds, preprint 2002.

[NR] Y. G. Nikonorov and E. D. Rodionov, Compact homogeneous Einstein 6-manifolds, preprint 2002.

[Pa] R. Palais, Critical point theory and the minimax principle, Proc. Symp. Pure Math. 15 (1970), 185-212.

[PS] J. S. Park and Y. Sakane, Invariant Einstein metrics on certain homogeneous spaces, Tokyo J. Math. 20 (1997), 51-60.

[Pe] J. Petean, The Yamabe invariant of simply connected manifolds, J. reine angew. Math. 523 (2000), 225231.

[Pe1] P. Petersen, Convergence theorems in Riemannian geometry, in Comparison Geometry, eds. K. Grove and P. Petersen, MSRI Publications 30 (1997), 167-202.

[Pe2] P. Petersen, Riemannian Geometry, Graduate Texts in Mathematics 171, Springer-Verlag (1997). 
[Ri] R. W. Richardson, Jr., A Rigidity theorem for subalgebras of Lie and associative algebras, Ill. J. Math. 14 (1967), 92-110.

[Rod] E. D. Rodionov, Einstein metrics on a class of five-dimensional homogeneous spaces, Comment. Math. Univ. Carolinae 32 (1991), 389-393.

[Roy] H. Royden, Real Analysis, Macmillan (1968).

[Sc1] R. Schoen, Conformal deformation of a Riemannian metric to constant scalar curvature, J. Diff. Geom. 20 (1984), 479-495.

[Sc2] R. Schoen, Variational theory for the total scalar curvature functional for Riemannian metrics and related topics, in Topics in the Calculus of Variations, Lecture Notes in Math. 1365 (1987), Springer-Verlag, 120154 .

[Tr] N. Trudinger, Remarks concerning the conformal deformation of Riemannian structures on compact manifolds, Ann. Scuola Norm. Sup. Pisa 22 (1968), 265-274.

[Wa] M. Wang, Einstein metrics from symmetry and bundle constructions, in Surveys in Differential Geometry VI: Essays on Einstein Manifolds, eds. C. LeBrun and M. Wang, International Press, (1999), 287-325.

[WZ1] M. Wang and W. Ziller, On the isotropy representation of a symmetric space, in Differential geometry on homogeneous spaces, Conf. Torino/Italy 1983, Rend. Sem. Mat. Torino, (1983), 253-261.

[WZ2] M. Wang and W. Ziller, Existence and non-existence of homogeneous Einstein metrics, Invent. Math. 84 (1986), 177-194.

[WZ3] M. Wang and W. Ziller, Einstein metrics on principal torus bundles, J. Diff. Geom. 31 (1990), 215-248.

[WZ4] M. Wang and W. Ziller, On isotropy irreducible Riemannian manifolds, Acta Math. 199 (1991), $223-261$.

[WZ5] M. Wang and W. Ziller, Primitive subgroups of finite-dimensional Lie groups, in preparation.

[Wh] H. Whitney, Elementary structure of real algebraic varieties, Ann. of Math. 66 (1957), 545-556.

[Wo1] J. Wolf, The geometry and structure of isotropy irreducible homogeneous spaces, Acta Math. 120 (1968), 59-148; correction: Acta Math. 152 (1984), 141-142.

[Wo2] J. Wolf, Spaces of Constant Curvature, 4th edition, Publish or Perish Inc., (1977).

[Ya] H. Yamabe, On a deformation of Riemannian structures on compact manifolds, Osaka Math. J. 12 (1960), 21-37.

[Zi] W. Ziller, Homogeneous Einstein metrics on spheres and projective spaces, Math. Ann. 259 (1982), 351358.

Universität Kiel, Kiel, Germany

E-mail address: boehm@math.uni-kiel.de

Mcmaster University, Hamilton, Ontario, Canada

E-mail address: wang@mcmaster.ca

University of Pennsylvania,Philadelphia, PA 19104,USA

E-mail address: wziller@math.upenn.edu 